\title{
LOWER BOUNDS ON THE COMPLEXITY OF POLYTOPE RANGE SEARCHING
}

\author{
BERNARD CHAZELLE
}

\section{INTRODUCTION}

Orthogonal range searching and simplex range searching have received much attention recently in the computational geometry literature. Whereas the former problem is nearing a definitive solution, however, the complexity of simplex range searching has long remained elusive. To state the problem simply, suppose that we are given $n$ points in Euclidean $d$-space, fixed once and for all, and $m$ units of computer memory. We wish to organize the memory to be in a position to answer the following type of queries efficiently: Given an arbitrary simplex $q$, how many of the $n$ points lie inside $q$ ? A natural variant of the problem calls for reporting the points in question and not simply counting them. More generally, it is customary to weight the points ahead of time and then ask for the cumulative weight of the subset of points that fall within the query. There is abundant practical application to motivate research on this problem $[5,6,7$, $10,11,15,18,20,22]$. For example, clipping and removing hidden surfaces in computer graphics are fundamental tasks whose computational bottlenecks are instances of simplex range searching. Also of great interest is the central theoretical question lying underneath: What is the most efficient way of organizing information to support a given class of queries? What takes this question apart from the classical problem of searching a linear list is the power of redundancy. While oversupply of memory space is usually of marginal interest when searching a linear list, it is often the key to efficiency in multidimensional searching. For this reason, the principal research activity in that area has been the investigation of space-time trade-offs.

Our main result is a family of lower bounds on the space-time complexity of simplex range searching. We prove that the worst case query time is $\Omega(n / \sqrt{m})$ in the Euclidean plane, and more generally, $\Omega\left((n / \log n) / m^{1 / d}\right)$ in $d$-space, for $d \geq 3$, where $n$ is the number of points and $m$ is the amount of storage available. ${ }^{1}$ These bounds hold with high probability for a random point-set

Received by the editors June 10, 1988 and, in revised form, April 26, 1989.

1980 Mathematics Subject Classification (1985 Revision). Primary 68U05, 68Q15.

The author wishes to acknowledge the National Science Foundation for supporting this research in part under Grant CCR-8700917.

A preliminary version of this work has appeared in the Proceedings of the 28th Annual IEEE Symposium on Foundations of Computer Science (1987), pp. 1-10.

${ }^{1}$ All algorithms in this paper are taken to the base 2 , unless specified otherwise. 
(from a uniform distribution in the unit $d$-cube) and thus are valid in the worst case as well as on the average. Interestingly, they still hold if the queries are restricted to congruent copies of a fixed simplex or even a fixed slab.

What is the practical significance of these lower bounds? The main lesson to be learned is that virtually no gain in query time can be expected unless we have close to unlimited storage. For example, in 11 -space, a query time as uninspiring as, say, $O(\sqrt{n})$ still requires at least on the order of $n^{5}$ storage. Our average case result makes matters even worse by saying that most input point-sets are hard, and not just some small pathological subset. In practice, therefore, the naive algorithm-which involves checking each of the $n$ points for inclusion individually-stands as the method of choice.

Our complexity results are established in the arithmetic model for range searching (Fredman [9, 10], Yao [19], Chazelle [4]). Briefly, a data structure in that model is a collection of precomputed values corresponding to the cumulative weights of certain subsets of the points. To answer a query involves adding together some of these weights. The model is tailored for lower bounds because the query time is made to reflect only how many weights must be added together to answer the query (hence the name "arithmetic") and not how long it takes to locate the needed information in memory. For this reason, any lower bound proven in the arithmetic model can be trusted to hold on any reasonable sequential machine (which, in particular, allows bucketing, hashing, etc.). How close do our lower bounds come to meeting known upper bounds? It has been shown (Chazelle and Welzl [5]) that simplex range searching on $n$ points in $d$ space can be performed in $O\left(n^{1-1 / d} \alpha(n)\right)$ query time and $O(n)$ storage, where $\alpha$ is a very slow-growing functional inverse of Ackermann's function. This upper bound, which holds in the arithmetic model, matches our lower bound very closely. On a random access machine [2] supplied with linear storage, the best upper bound on the query time to date is $O(\sqrt{n} \log n)$ in 2-space (Chazelle and Welzl [5]) and $O\left(n^{d(d-1) /(d(d-1)+1)+\varepsilon}\right)$ in $d$-space, for any $d \geq 3$ and any fixed $\varepsilon>0$ (Haussler and Welzl [11]). A query time of $O\left(n^{2 / 3} \log ^{2} n\right)$ can be achieved in the three-dimensional case, if $O(n \log n)$ storage is available [5]. We also refer the reader to Willard [18], Edelsbrunner and Welzl [7], and Cole and Yap [6] for earlier results on the problem and variants of it.

Our results constitute the first (nontrivial) family of lower bounds for simplex range searching in the static case. These complement an earlier lower bound for the dynamic version of the problem: Fredman [10] established that a sequence of $n$ insertions, deletions, and half-plane range queries may require $\Omega\left(n^{4 / 3}\right)$ time. His ingenious proof technique rests on the fact that a single deletion may invalidate a large segment of the data structure. Indeed, any precomputed cumulative weight which involves a point to be deleted becomes useless after the deletion, since a semigroup has no inverse and no quick update is therefore possible. Interestingly, our lower bound can be used to strengthen Fredman's result by removing the need for deletions. Indeed, we can exhibit a sequence of $n$ insertions, followed by $n$ queries which, together, require at least on the order of $n^{4 / 3}$ time. To see this, set $m=n^{4 / 3}$ and apply our two-dimensional lower bound. It states the existence of a set $P$ of $n$ points which no data structure of 
size $m$ can preserve from a query of cost $\Omega(n / \sqrt{m})=\Omega\left(n^{1 / 3}\right)$. So, insert each point of $P$ one at a time and then ask the hardest query $n$ times. Since many cumulative weights might be computed between successive queries, we will not necessarily be asking the same query all the time; however, we will always ask the hardest query in light of all precomputed information so far. If the total running time exceeds $n^{4 / 3}$, then our point is made. Otherwise, the algorithm does not have the time to compute a data structure of size in excess of $n^{4 / 3}$ (up to within constant factors). Therefore, each query will cost $\Omega\left(n^{1 / 3}\right)$, which will bring the total running time to $\Omega\left(n^{4 / 3}\right)$. In dimension $d>2$, we have the result that $n$ insertions followed by $n$ queries cost at least on the order of $\left(n^{2} / \log n\right)^{d /(d+1)}$ time in the worst case.

Returning to the main lower bounds of this paper, our approach is to reduce space-time trade-offs for range searching to certain inequalities in integral geometry. To achieve this goal we need some machinery which we build in three main stages. First, we define a model for static range searching $(\S 2)$ which places the problem within the scope of bipartite Ramsey theory $(\S 3)$. The complexity of a given problem is then fully described by certain properties of its so-called characteristic graph. This involves two distinct tasks: proving integral-geometric inequalities about the query space $(\S 4.2)$ and studying various uniformity criteria for random point-sets $(\S 4.3)$. Incidentally, these investigations lead to results of independent interest regarding an intriguing generalization of Heilbronn's problem (Moser [16]). Briefly, the problem in two dimensions is this: Given two integers $n$ and $k \leq n$, place $n$ points in a unit square so that the convex hull of any $k$ of them has an area at least $c k / n$, for some fixed constant $c>0$. We show that this can be done if $k$ exceeds $\log n$. This result completes the set of tools needed to prove the lower bounds for simplex range searching $(\S 4.4)$.

\section{A COMBINATORIAL FRAMEWORK}

We describe a graph-theoretic model for range searching. The emphasis of this model is the arithmetic complexity of a problem, that is, the maximum number of operations needed to answer any query. The model purposely ignores the cost of searching the memory for the information needed during the computation. In this way, lower bounds can be trusted to hold on any sequential computer. Of course, from a practical viewpoint, upper bounds set in that model may not necessarily have much meaning, except to indicate how good or how bad a certain lower bound might be. The arithmetic model-as it is customarily called-originates in Fredman $[9,10]$ for the dynamic case and Yao [19] for the static case.

The main purpose of this section is to introduce a general technique for proving lower bounds (the Core Lemma). The basic idea is to relate the static complexity of a range searching problem to the existence of large complete bipartite subgraphs in its characteristic graph. This graph provides a combinatorial characterization of a range searching problem. 
A. Some terminology. In the following, $\mathscr{N}$ will denote the set of natural numbers $\{0,1,2, \ldots\}$, and for any integer $n>0,[1 \ldots n]$ will be the set $\{1,2$, $\ldots, n\}$. We use $\mathscr{C}_{d}$ to denote the unit $d$-cube $[0,1]^{d}$. As a shorthand, we say that a finite set of points $P$ in a compact set $K$ is random in $K$ if each of its points has been drawn randomly from a uniform distribution in $K$ (we assume mutual independence). In general, $K$ will be $\mathscr{C}_{d}$. Finally, we introduce the notion of a faithful semigroup (Yao [19]). Let $(S,+)$ be a commutative semigroup with an operation denoted + . We say that $(S,+)$ is faithful if for each $n>0, \varnothing \subset T_{1}, T_{2} \subseteq[1 \ldots n], T_{1} \neq T_{2}$, and every sequence of integers $\alpha_{i}, \beta_{j}>0 \quad\left(i \in T_{1}, j \in T_{2}\right)$, there exists an assignment of semigroup values to the variables $s_{1}, \ldots, s_{n}$, such that

$$
\sum_{i \in T_{1}} \alpha_{i} s_{i} \neq \sum_{j \in T_{2}} \beta_{j} s_{j} .
$$

Note that this definition does not prohibit idempotence or more general identities, e.g., $s_{1}+2 s_{2}=3 s_{1}+4 s_{2}$. For example, $(\mathcal{N},+),(\mathscr{N}, \max )$, and $(\{0,1\}$, or) are faithful, but $(\{0\}$, or $)$ and $(\{0,1\}$, exclusive-or $)$ are not.

B. Range searching. Let $(S,+)$ be a faithful commutative semigroup. We define a query space $\mathscr{Q}$ to be any collection (finite or infinite) of subsets $q \subseteq \mathfrak{R}^{d}$, called queries. For example, $\mathscr{Q}$ might be the set of all hyperrectangles, simplices, balls in Euclidean $d$-space, etc. Let $P=\left\{p_{1}, \ldots, p_{n}\right\}$ be a set of $n$ points in $\mathscr{C}_{d}$, and let $\zeta$ (the weight function) be an assignment of each point $p_{i}$ to a semigroup value in $S$. We define a function answ: $\mathscr{Q} \rightarrow S$ as follows:

$$
\operatorname{answ}(q)=\sum_{p \in P \cap q} \zeta(p) \text {. }
$$

If $P \cap q$ is empty, then we write answ $(q)=$ null, which is a special symbol not in $S$. In practice, the semigroup can be chosen as $(\mathcal{N},+)$ for counting the number of points in the desired query, $\left(2^{P}, \cup\right)$ for reporting the points in question, $(\{0,1\}$, or $)$ for testing if there are any points in the query, etc. To summarize, a range searching problem $\mathscr{P}$ is specified by a quadruple $(S, \mathscr{Q}, P, \zeta)$ consisting of a semigroup, a query space, a finite set of points, and a weight function. We say that $\mathscr{P}$ is of size $(n, p)$ if $|P|=n$ and $|\{P \cap q \mid q \in \mathscr{Q}\}|=p$.

C. The model of computation. Let $s_{1}, \ldots, s_{n}$ be $n$ variables with values in $S$. A generator $g\left(s_{1}, \ldots, s_{n}\right)$ is a linear form $\sum_{1 \leq i \leq n} \alpha_{i} s_{i}$, where the $\alpha_{i}$ 's are nonnegative integers (not all 0 ). For example, $2 s_{1}+0 s_{2}+s_{3}$ stands for $s_{1}+s_{1}+s_{3}$. A storage scheme $\Gamma$ for $\mathscr{P}$ of size $m$ is a collection of $m$ generators $\left\{g_{1}, \ldots, g_{m}\right\}$ satisfying the following property. For any $q \in \mathscr{Q}$ such that $P \cap q \neq \varnothing$, there exist $K \subseteq[1 \ldots m]$ and a set of labeled integers $\left\{\beta_{k}>0 \mid k \in K\right\}$ such that the relation

$$
\operatorname{answ}(q)=\sum_{k \in K} \beta_{k} g_{k}\left(\zeta\left(p_{1}\right), \ldots, \zeta\left(p_{n}\right)\right)
$$

holds for any weight function $\zeta$ over $P$. This means that a storage scheme can be dependent on the particular semigroup under consideration and also take 
advantage of any property which $P$ may enjoy; however, it must hold for any assignment of semigroup values to $P$. This last point is important. It implies that our lower bounds do not apply to, say, counting the number of points inside a query simplex. Indeed, a data structure for this problem need work only for the particular weight assignment $\zeta(p)=1$.

Ideally, we would like $S$ to be rich enough to simulate (i.e., to map homomorphically onto) the semigroup $\left(P^{*}, \cup\right)$ of all nonempty subsets of $P$. But this would exclude too many important semigroups, so we move this requirement over to the storage scheme. By insisting that a scheme should work for all weight assignments, we are in effect no longer dealing with $S$ itself but with the additive semigroup of $n$-variate linear forms over $S$. Faithfulness can then be called upon to ensure that the semigroup of linear forms is, indeed, rich enough. Given a linear form $\sum_{1 \leq i \leq n} \alpha_{i} s_{i}$, call the set of points $\left\{p_{i} \mid \alpha_{i} \neq 0\right\}$ its cluster. ${ }^{2}$ By means of this correspondence, the semigroup generated by the elementary forms $\left(s_{1}, \ldots, s_{n}\right) \mapsto s_{i} \quad(1 \leq i \leq n)$ maps homomorphically onto the semigroup $\left(P^{*}, \cup\right)$. Thus, the meaning of $(2.1)$ is that any set of the form $P \cap q$ can be expressed as a union of clusters; the union need not be disjoint. Note that the basic irrelevance of the weight function allows us to say that a storage scheme is defined not only with respect to $\mathscr{P}$, but more generally, with respect to the triple $(S, \mathscr{Q}, P)$.

Next, we define the complexity of a storage scheme $\Gamma$ with $m$ generators. Given $q \in \mathscr{Q}$, let $K$ be the smallest subset of generator indices such that $(2.1)$ is true. We define $t(P, \Gamma, q)=|K|$, and we say that $\Gamma$ is a $(t, m)$-scheme for $\mathscr{P}$, if $t \geq \max _{q \in \mathscr{Q}} t(P, \Gamma, q)$. If $\mathscr{P}$ is now considered as one element in an infinite family (as $P$ and $n$ vary), we define the time complexity of this family as the function $t(n, m)$, where

$$
t(n, m)=\max _{|P|=n} \min _{|\Gamma|=m} \max _{q \in \mathscr{Q}} t(P, \Gamma, q) \text {. }
$$

By abuse of notation, we will refer to $t(n, m)$ as the time complexity of $\mathscr{P}$ (when the notion of a family is understood). We also define the expected time complexity of $\mathscr{P}$ as

$$
\bar{t}(n, m)=E_{|P|=n} \min _{|\Gamma|=m} \max _{q \in \mathscr{Q}} t(P, \Gamma, q),
$$

where $P$ is random in $\mathscr{C}_{d}$. We do not average over $\mathscr{Q}$ because the query space cannot always be assumed to admit a natural probability measure.

D. The graph model. We begin with some terminology. Let $H \subseteq V \times W$ be a bipartite graph. We denote the number of edges of $H$ by $|H|$. For any $w \in W$, let $N_{H}(w)$ denote the neighbor set $\{v \in V \mid(v, w) \in H\}$. By extension, if $U \subseteq W$ then $N_{H}(U)=\bigcup_{w \in U} N_{H}(w)$. Given an arbitrary set $Z$, a bipartite graph $C \subseteq V \times Z$ is called a cover of $H$ if for every $w \in W$ there exists a subset $Z_{w} \subseteq Z$ such that $N_{H}(w)=N_{C}\left(Z_{w}\right)$. A subset $Z_{w}$ of minimum size is called a min-cover of $w$. If the cardinality of no min-cover exceeds $t$, we say

\footnotetext{
${ }^{2}$ To make this definition independent of the fact that $S$ is faithful, we should regard a cluster as being associated with a formal linear form. Otherwise, a cluster is not uniquely defined if $S$ is not faithful and a linear form can be expressed over two different set of variables.
} 
that $C$ is a $(t,|Z|)$-cover of $H$. The graph $C$ is called a disjoint $(t,|Z|)$-cover if

(i) for each $w \in W$, there exists $Z_{w} \subseteq Z$ such that $N_{H}(w)=N_{C}\left(Z_{w}\right)$ and all the sets in $\left\{N_{C}(z) \mid z \in Z_{w}\right\}$ are pairwise disjoint, and

(ii) the maximum value, over all $w \in W$, of the size of the smallest $Z_{w}$ satisfying (i) does not exceed $t$.

In light of our discussion of generators and clusters the meaning of all this should be obvious. We can use $H$ to model a range searching problem, with the $V$ nodes acting as points and the $W$-nodes as queries. A cover $C$ corresponds to a storage scheme, with the $Z$-nodes acting as generators and the $N_{C}(z)$ 's $(z \in Z)$ as clusters. To conclude this string of definitions, we call a rectangle of $H$ any complete bipartite subgraph $V^{\prime} \times W^{\prime} \subseteq H$; the width and height of the rectangle are, respectively, $\left|V^{\prime}\right|$ and $\left|W^{\prime}\right|$.

Given a range searching problem $\mathscr{P}=(S, \mathscr{Q}, P, \zeta)$ of size $(n, p)$, the set $\{P \cap q \mid q \in \mathscr{Q}\}$ partitions $\mathscr{Q}$ into $p$ equivalence classes. Let $q_{1}, \ldots, q_{p}$ be representatives of each class. We define the characteristic graph of $\mathscr{P}$ as a bipartite graph $H \subseteq V \times W$, where $V=\left\{v_{1}, \ldots, v_{n}\right\}$ and $W=\left\{w_{1}, \ldots, w_{p}\right\}$, and an edge connects $v_{i}$ and $w_{j}$ if and only if $p_{i} \in q_{j}$. The graph fully describes the combinatorial nature of the range searching problem in question. Conversely, any bipartite graph can be regarded as the characteristic graph of some reasonably natural range searching problem: for example, $P$ might consist of $n$ distinct points in the plane, with $\mathscr{Q}$ defined as the set of polygonal curves. We are now in a position to formalize the relationship between schemes and covers, and present a methodology for proving space-time trade-offs. Generators are to schemes what clusters are to covers. The following lemma uses faithfulness to establish that link.

Lemma 2.1. Let $\mathscr{P}$ be a range searching problem, and let $H$ be its characteristic graph. If $\mathscr{P}$ admits a $(t, m)$-scheme, then $H$ admits $a(t, m)$-cover. Conversely, if $H$ admits a disjoint $(t, m)$-cover, then $\mathscr{P}$ admits $a(t, m)$-scheme.

Proof. Suppose that $\mathscr{P}$ admits a $(t, m)$-scheme, and let $\Gamma=\left\{g_{1}, \ldots, g_{m}\right\}$ be the storage scheme in question. If $g_{k}\left(s_{1}, \ldots, s_{n}\right)=\sum_{1 \leq i \leq n} \alpha_{k, i} s_{i}$, we can rewrite this linear form as $\sum_{i \in N_{k}} \alpha_{k, i} s_{i}$, where $N_{k}=\left\{i \mid \alpha_{k, i}>0\right\}$. We define a bipartite graph $C \subseteq V \times Z$, where $Z=\left\{z_{1}, \ldots, z_{m}\right\}$, by placing an edge between $v_{i}$ and $z_{j}$ if and only if $i \in N_{j}$. We now show that $C$ is a $(t, m)$ cover of $H$. Let $w_{j}$ be an arbitrary vertex of $W$, and let $A_{j}=\left\{i \mid p_{i} \in q_{j}\right\}$. It suffices to establish the existence of $Z_{j} \subseteq Z$, where $\left|Z_{j}\right| \leq t$ and

$$
N_{H}\left(w_{j}\right)=N_{C}\left(Z_{j}\right)
$$

Since $\Gamma$ is a $(t, m)$-scheme for $P$, we have

$$
\operatorname{answ}\left(q_{j}\right)=\sum_{k \in B_{j}} \beta_{k} g_{k}\left(\zeta\left(p_{1}\right), \ldots, \zeta\left(p_{n}\right)\right),
$$


where $B_{j}$ is a subset of $[1 \cdots m]$ of size $\leq t$. Since the equality above holds for any weight function and $S$ is a commutative semigroup, we can write

$$
\sum_{i \in A_{j}} s_{i}=\sum_{k \in B_{j}} \beta_{k} g_{k}\left(s_{1}, \ldots, s_{n}\right)=\sum_{k \in C_{j}} \gamma_{k} s_{k},
$$

where $C_{j}=\bigcup_{k \in B_{j}} N_{k}$. Because of faithfulness we have $A_{j}=C_{j}$; therefore,

$$
N_{H}\left(w_{j}\right)=\left\{v_{i} \mid i \in A_{j}\right\}=\bigcup_{k \in B_{j}}\left\{v_{i} \mid i \in N_{k}\right\}=\bigcup_{k \in B_{j}} N_{C}\left(z_{k}\right)
$$

which establishes (2.2), since $\left|B_{j}\right| \leq t$. The first part of the lemma is now proven. We omit the second part, which is straightforward.

Now that range searching problems have been couched as combinatorial questions about bipartite graphs, we are ready to describe the lower bound proof technique which underlies much of what follows. Although the technique tends to weaken somewhat on problems of low complexity (e.g., orthogonal range queries), it is, we believe, a powerful tool for determining the complexity of "hard" problems, such as simplex range searching or problems defined by random characteristic graphs. The starting point is the observation that, informally, clusters are "good" if they are big and can be used to answer many representative queries. Translated in the language of covers, this means that for problems of low complexity the characteristic graph must contain many rectangles of large "area"; their widths tell us how big the clusters can be and their heights indicate how many representative queries they can help to answer. Thus, lower bounds are obtained by proving that rectangles can never be too large.

The following result formalizes the relationship between the space complexity of a range searching problem $\mathscr{P}$ and the presence of large rectangles in its characteristic graph $H$. We define $\mathscr{A}(x)$ to be the largest "area" of a rectangle of $H$ whose width is no less than $x>0$ :

$$
\mathscr{A}(x)=\max \{x h \mid H \text { has a rectangle of width } \geq x \text { and height } h\} .
$$

Lemma 2.2 (The Core Lemma). Let $H$ be the characteristic graph of a range searching problem of size $(n, p)$. If $H$ has a $(t, m)$-cover, then $m \geq$ $\frac{1}{2}|H| / \mathscr{A}(|H| / 2 p t)$.

Proof. Using the previous notation, let $C \subseteq V \times Z$ be a $(t, m)$-cover of $H$ and let $\left\{C_{w} \subseteq Z \mid w \in W\right\}$ be a complete collection of min-covers. Form the graph $G$ by removing from $H$ each edge in the set

$$
\bigcup\left\{N_{C}(z) \times\{w\}\left|w \in W, \quad z \in C_{w},\right| N_{C}(z) \mid \leq \frac{|H|}{2 p t}\right\} .
$$

Since $C$ is a $(t, m)$-scheme and $|W|=p$, at most $p t|H| /(2 p t)$ edges are removed, and so the resulting graph $G$ contains at least half the edges of $H$. But to cover the sets $N_{G}(w) \quad(w \in W)$, only subsets $N_{C}(z)$ of size $>|H| / 2 p t$ are now used. Therefore $Z$ must have at least $|G| / \mathscr{A}(|H| /(2 p t))$ vertices.

\section{HOW HARD CAN RANGE SEARCHING BE?}

Any range searching problem of size $(n, p)$ admits two trivial solutions: an $(n, n)$-scheme and a $(1, p)$-scheme. Two natural questions arise: (1) Is 
it always possible to improve over the two naive solutions? (2) What is the complexity of the hardest range searching problem? Answering these questions will help us assess the relative position of other range searching problems on the complexity ladder. Theorem 3.1 says that a small speed-up in query time can always be achieved with an amount of storage almost but not quite maximum, in other words, the worst of all possible worlds. Surprisingly, this result is in fact optimal, as we can show by a probabilistic argument (Theorem 3.2).

Theorem 3.1. For any range searching problem of size $(n, p)$, with $p>n$, there exists a $(t, m)$-scheme, where $t=O(n /\lceil\log (p / n)\rceil)$ and $m=O(p /\lceil\log (p / n)\rceil)$. Proof. We follow a strategy used in Yao and Yao [21] and Burkhard et al. [3]. Let $\alpha=\lceil\log (p / n)\rceil$ and, as usual, let $H \subseteq V \times W$ denote the characteristic graph of the range searching problem, with $V=\left\{v_{1}, \ldots, v_{n}\right\}$. For each $i$ such that $0 \leq i \leq\lfloor(n-1) / \alpha\rfloor$, define $V_{i}$ as the set $\left\{v_{i \alpha+1}, \ldots, v_{\min \{n,(i+1) \alpha\}}\right\}$. We construct a cover $C \subseteq V \times Z$ as follows. Originally, $Z$ is empty; for each $i$ between 0 and $\lfloor(n-1) / \alpha\rfloor$, consider each nonempty subset $A$ of $V_{i}$ in turn, and perform the following operations: add a new vertex $z$ to $Z$ and augment $C$ with the edges of $A \times\{z\}$. It is easily verified that $C$ is a disjoint $(t, m)$-cover of $H$, where $t \leq 1+\lfloor(n-1) / \alpha\rfloor$, and

$$
m \leq\left(2^{\alpha}-1\right)(1+\lfloor(n-1) / \alpha\rfloor) \text {. }
$$

Since $p \leq 2^{n}$, we easily derive that $t=O(n /\lceil\log (p / n)\rceil)$ and that $m=$ $O(p /\lceil\log (p / n)\rceil)$. Lemma 2.1 completes the proof.

Theorem 3.2. There is a constant $c>0$ such that the following is true. Given any integer function $p=p(n) \quad\left(n<p \leq 2^{n}\right)$ there exists a class of range searching problems of size $(n, p)$ for which any $(t, m)$-scheme with $t \leq c n / \log (p / n)$ also satisfies $m=\Omega(p)$.

Proof. Let $\pi$ be a real $(0<\pi<1)$, and let $H \subseteq V \times W$ be a random bipartite graph $(|V|=n$ and $|W|=p)$, where each edge $(v, w)$ is chosen independently with probability $\pi$. A rectangle of $H$ is called wide if its width $\alpha$ is at least $\ln (p / n)$ and its height is equal to $\lceil n / \alpha\rceil$. To rid the graph of wide rectangles, we use a standard technique for removing forbidden subsystems (Erdös and Spencer [8]). Let $\chi(H)$ be the number of wide rectangles in $H$. We modify $H$ by taking each wide rectangle in turn, and removing exactly one edge from it (which one does not matter). After at most $\chi(H)$ such operations we obtain a new graph $G$ free of wide rectangles, with $|G| \geq|H|-\chi(H)$. Taking expectations we derive

$$
E(|G|) \geq n p \pi-\sum_{\ln (p / n) \leq \alpha \leq n}\left(\begin{array}{l}
n \\
\alpha
\end{array}\right)\left(\begin{array}{c}
p \\
\lceil n / \alpha\rceil
\end{array}\right) \pi^{\alpha\lceil n / \alpha\rceil} .
$$

Using the inequalities $\left(\begin{array}{l}b \\ a\end{array}\right)<(e b / a)^{a}$, for $0<a \leq b$, and $(n / \alpha)^{\alpha} \leq e^{n / e}$, for $1 \leq \alpha \leq n$, where $e=2.718 \cdots$, we derive that for $n$ large enough,

$$
\left(\begin{array}{l}
n \\
\alpha
\end{array}\right)\left(\begin{array}{c}
p \\
\lceil n / \alpha\rceil
\end{array}\right) \pi^{\alpha\lceil n / \alpha\rceil}<\left(\frac{p \alpha}{n}\right)^{2 n / \alpha} \pi^{n} e^{\alpha+\lceil n / \alpha\rceil+n / e}<e^{(4+(2 / \alpha) \ln (p / n)+\ln \pi) n} .
$$

If $\pi=e^{-6}$, it then follows that for $n$ large enough,

$$
E(|G|)>n p \pi-n e^{(6+\ln \pi) n}>n p / e^{7},
$$


so there exists a bipartite graph $G \subseteq V \times W$, with at least $n p / e^{7}$ edges and no wide rectangle. With respect to this graph, we have $\mathscr{A}(x)<2 n$, for $x \geq \ln (p / n)$, so from the Core Lemma, any $(t, m)$-cover such that $t \leq$ $c(n / \log (p / n))$ will satisfy $m>p / e^{9}$, for $c$ small enough. From Lemma 2.1 and our earlier observation that a range searching problem can always be defined to have a prespecified characteristic graph, the proof is now complete.

The comparison between the last two theorems is a little startling. On the one hand, for $p$ large enough, a time speed-up is always possible without using maximum storage. However, trying to improve this speed-up by even a constant factor will immediately force upon us the use of maximum storage (up to within a constant factor). The conclusion to draw is that, in practice, hard range searching problems do not offer any viable alternative to the two naive algorithms.

Remark. There is an intriguing parallel between this result and an equally pessimistic trade-off between update and query time given in Burkhard et al. [3]. Roughly speaking, the trade-off says that for some range searching problems any attempt to update weights faster than the naive way will cause a dramatic rise in the query time. Of course, the two situations cannot really be compared, however, because of the difference in settings: storage vs. query time here, as opposed to update time vs. query time in [3]. Without pursuing this digression too far, let us point out just one major difference between the static and the dynamic models. In the former, a cluster is charged unit cost, regardless of its size. In the dynamic model, however, a large cluster, although still charged unit cost, is in effect more costly than a small one because it is more exposed to enemy fire: if any of its points is updated the information provided by the cluster must be thrown away.

\section{THE COMPLEXITY OF SIMPLEX RANGE SEARCHING}

We begin by stating the main result of this section: simplex range searching on $n$ points requires $\Omega(n / \sqrt{m})$ query time in two dimensions and $\Omega\left((n / \log n) / m^{1 / d}\right)$ query time in any dimension $d \geq 3$. These bounds hold for a random point-set (uniform distribution in the unit $d$-cube) with high probability, and thus are valid in the worst case as well as on the average.

For technical reasons, queries will be slabs of fixed width instead of simplices (since slabs can always be clipped and triangulated, this will actually strengthen our results). The heart of the argument comes from the Core Lemma: a generator can be very useful to a small subset of all possible queries or it can be moderately useful to a large set of queries, but it cannot be very useful to lots of queries. We assess the "effectiveness" of a generator by the Lebesgue measure of the convex hull of its associated cluster. Why? Suppose that we set our sights on a very low query time. Then, presumably, to answer a random query requires the use of big clusters. Since the points are uniformly distributed in $\mathscr{C}_{d}$, big clusters occupy a lot of space and therefore can be used by only a small set of queries. This suggests a trade-off between the effectiveness of a generator and its ability to be used by many queries. One will notice the similarity of this reasoning with the Core Lemma. 
Our approach has two components. We begin with an integral-geometric analysis of the containment property between a convex body and a slab. The goal is to produce a continuous analog of the discrete complexity trade-off sought. To carry out the analogy we must be able to place $n$ points in the unit $d$-cube so that the cardinality of any subset is at most proportional to $n$ times the measure of its convex hull. This entails a study of pseudouniform point-sets. The questions raised are akin to a classical problem of Heilbronn (Moser [16]) to which we provide new answers.

In $\S 4.1$ we define a measure for slab systems, and we prove its invariance under the group of motions. This will give us a convenient probability measure for queries to work with. In $\S 4.2$ we argue that a large convex set cannot be moved too much within a given slab (in other words, a big cluster cannot be used by too many queries). Two fundamental lemmas are derived to formalize this concept. In $\S 4.3$ we turn to the problem of approximating uniform point distributions. Several criteria of uniformity are investigated, one of which leads to new results on a generalization of Heilbronn's problem. Finally $\S 4.4$ puts all the above results together and derives the desired lower bounds.

4.1. Preliminaries. We begin with some geometric terminology. Let $d$ be a fixed positive integer, and let $E^{d}$ denote Euclidean $d$-space. Unless specified otherwise, we will always assume that $d>1$. We endow $E^{d}$ with a Cartesian system of reference $\left(O, \mathbf{e}_{1}, \ldots, \mathbf{e}_{d}\right)$, where $\left(\mathbf{e}_{1}, \ldots, \mathbf{e}_{d}\right)$ forms an orthonormal basis $\left(\mathbf{e}_{i} \cdot \mathbf{e}_{j}=\delta_{i j}\right)$. We define $E_{+}^{d}=\left\{\left(x_{1}, \ldots, x_{d}\right) \neq O \mid x_{i} \geq 0 ; 1 \leq i \leq d\right\}$. If $p=\left(x_{1}, \ldots, x_{d}\right)$ and $q=\left(y_{1}, \ldots, y_{d}\right)$ are two points of $E^{d}$, then we let $\langle p, q\rangle$ denote the inner product $\sum_{1 \leq i \leq d} x_{i} y_{i}$. Similarly, we put $|p|=\sqrt{\langle p, p\rangle}$.

The width (resp. diameter) of a compact convex set $K$ is the smallest (resp. largest) distance between two distinct hyperplanes of support parallel to each other. The diameter of $K$ is denoted $D(K)$. It is also defined as the greatest distance between any pair of points in $K$. Finally, if $P$ is a finite set of points in $E^{d}$, then $\kappa(P)$ denotes its convex hull.

Let $\alpha$ be a real value $(0<\alpha<1 / 12)$ to be considered a parameter in the following. We define a slab as the closed region of $E^{d}$ between any pair of parallel hyperplanes distant from each other by $2 \alpha$. For any $q \in E^{d} \backslash\{O\}$, let $S_{q}$ denote the slab

$$
S_{q}=\left\{p \in E^{d}:\left.|\langle p, q\rangle-| q\right|^{2}|\leq \alpha| q \mid\right\} .
$$

We use slabs as queries, instead of simplices, because they are easier to manipulate. Straightforward reductions will show that this does not artificially increase the complexity of the problem (if anything, it makes the problem easier). Using the notation of the exterior calculus, it is well known (Santalo [17]) that the point-set density $d x_{1} \wedge d x_{2} \wedge \cdots \wedge d x_{d}$ is invariant under the group of motions (i.e., isometries). Given $X \subset E^{d}$, the integral

$$
\lambda_{d}(X)=\int_{X} d x_{1} \wedge \cdots \wedge d x_{d}
$$


is the measure of $X$ in $E^{d}$ (provided that the integral exists in the Lebesgue sense). Next we define the measure $\mu$ of a set of slabs $X$ :

$$
\mu(X)=\int_{X} d S_{q}=\int_{S_{q} \in X} \frac{d y_{1} \wedge \cdots \wedge d y_{d}}{|q|^{d-1}},
$$

where $q=\left(y_{1}, \ldots, y_{d}\right)$ (again, provided that the integral exists). Since $S_{q}$ is not defined for $q=O$, we may assume that $X$ does not contain slabs whose bisecting hyperplanes pass through the origin. This is not necessary, however, because the integral

$$
\int_{0 \leq|q|<t} \frac{d y_{1} \wedge \cdots \wedge d y_{d}}{|q|^{d-1}}
$$

is well defined and tends to 0 as $t \rightarrow 0$. (The set of slabs whose bisecting planes pass through the origin has measure zero.) Our choice of measure is motivated by the following.

Lemma 4.1. The measure $\mu$ is invariant under the group of isometries in $E^{d}$.

Proof. We use the techniques of Santaló [17], and in particular, Cartan's method of moving frames. Let $P_{q}=\left\{\left.p \in E^{d}|\langle p, q\rangle=| q\right|^{2}\right\}$ be the bisecting hyperplane of $S_{q}$, and let $\mathbf{u}_{1}, \ldots, \mathbf{u}_{d-1}$ be an orthonormal basis for $P_{q}$. We define $\mathbf{u}_{d}$ as a unit vector normal to $P_{q}$ such that $\operatorname{det}\left(\mathbf{u}_{1}, \ldots, \mathbf{u}_{d}\right)=1$. Let $\mathscr{M}$ be the group of motions in $E^{d}$, and let $\mathfrak{F}$ be the subgroup of motions that leave invariant the hyperplane $P_{q}$. We have a one-to-one correspondence between the hyperplanes of $E^{d}$ and the elements of the homogeneous space $\mathscr{M} / \mathfrak{F}=\{g \mathfrak{F} \mid g \in \mathscr{M}\}:$ to each coset of the form $g \mathfrak{F}(g \in \mathscr{M})$ corresponds the hyperplane $g P_{q}$, and conversely, to each hyperplane $P_{r}$ corresponds the coset $g \mathfrak{F}$, where $g$ is a motion that carries $P_{q}$ to $P_{r}$. Following [17], finding an invariant density for hyperplanes, and hence for slabs, is then reduced to finding an invariant density $d L_{d-1}$ on $\mathscr{M} / \mathfrak{F}$. The theory of moving frames gives us the Pfaffian system $d_{q} \cdot \mathbf{u}_{d}=0$ and $d \mathbf{u}_{d} \cdot \mathbf{u}_{i}=0 \quad(1 \leq i \leq d-1)$. Therefore, $d L_{d-1}$ can be chosen as the differential exterior $d$-form

$$
\bigwedge_{i<d} d \mathbf{u}_{d} \cdot \mathbf{u}_{i} \wedge d q \cdot \mathbf{u}_{d} .
$$

Let $q=\left(y_{1}, \ldots, y_{d}\right)$. Since $\mathbf{u}_{d}=q /|q|$, we have $d \mathbf{u}_{d}=d q /|q|+d(1 /|q|) q$. From $\mathbf{u}_{i} \cdot \mathbf{u}_{j}=\delta_{i j}$ we derive

$$
\begin{aligned}
d L_{d-1} & =\bigwedge_{i<d}\left(\frac{1}{|q|} d q \cdot \mathbf{u}_{i}\right) \wedge d q \cdot \mathbf{u}_{d}=\frac{1}{|q|^{d-1}} \bigwedge_{i \leq d} \sum_{1 \leq j \leq d} d y_{j}\left(\mathbf{u}_{i} \cdot \mathbf{e}_{j}\right) \\
& =\frac{\Delta}{|q|^{d-1}} d y_{1} \wedge \cdots \wedge d y_{d}
\end{aligned}
$$

where

$$
\Delta=\operatorname{det}\left(\mathbf{u}_{1}, \ldots, \mathbf{u}_{d}\right)=1 .
$$

This establishes that $d L_{d-1}=\left(1 /|q|^{d-1}\right) d y_{1} \wedge \cdots \wedge d y_{d}$, which is precisely the differential form claimed for $\mu$. 
Now that we have the appropriate tools to handle random queries, we need some machinery to study the convex hull of clusters. Arbitrary convex subsets of $\mathscr{C}_{d}$ are a little unwieldy. Fortunately, they can be approximated quite well by rectangular shapes. We need some additional terminology. A hyperrectangle is the Cartesian product of $d$ closed intervals in $\mathfrak{R}$. If each interval is of the same length, then we have a hypercube. A parallelotope is the image of a hyperrectangle under an isometric mapping. Two parallelotopes are said to be parallel to each other if they are congruent modulo a homothetic transformation. It seems that the following equivalence result has been rediscovered many times over the years. (I thank J. Pach for pointing out this 1951 reference (Macbeath [14]) to me.)

Lemma 4.2 (Macbeath, 1951). Given a compact convex set $K$ in $E^{d}$, there exist two parallelotopes $\Pi_{1}$ and $\Pi_{2}$, such that $\Pi_{1} \subseteq K \subseteq \Pi_{2}$ and $\lambda_{d}\left(\Pi_{2}\right) / d ! \leq$ $\lambda_{d}(K) \leq d^{d} \lambda_{d}\left(\Pi_{1}\right)$.

4.2. Two fundamental lemmas on the measure of slabs. Let $K$ be an arbitrary compact convex subset of $E^{d}$. Our main concern in this section is to show that the set of slabs

$$
H(K)=\left\{S_{q} \mid q \in E^{d} \backslash\{O\} \text { and } K \subseteq S_{q}\right\}
$$

shrinks fast enough as $K$ grows. We will distinguish between the general case (Lemma 4.5) and a rather special case (Lemma 4.6) to be used later for simplex range searching in 2-space. The reason for this distinction is that we can obtain sharper lower bounds in the two-dimensional case by using more refined tools.

We begin our investigation by assuming that $K$ is a hyperrectangle of the form $\prod_{1 \leq i \leq d}\left[1, \gamma_{i}\right]$, where $\gamma_{1}, \ldots, \gamma_{d}$ are $d$ reals $\geq 1$. We will simplify our study of $H(K)$ by considering the smaller set

$$
H^{+}(K)=H(K) \cap\left\{S_{q} \mid q \in E_{+}^{d}\right\} .
$$

This substitution is fairly innocuous, as the following result shows.

Lemma 4.3. For any hyperrectangle $K=\prod_{1 \leq i \leq d}\left[1, \gamma_{i}\right]$, where $\gamma_{i} \geq 1$, we have the inequality $\mu(H(K)) \leq 2^{d} \mu\left(H^{+}(K)\right)$.

Proof. The idea is to consider the symmetry group of the polytope $K$ and identify $2^{d}$ automorphisms, at least one of which maps any given point of $E^{d}$ to a point with nonnegative coordinates. The proof will immediately follow from the fact that these automorphisms carry $K$ into itself and the measure $\mu$ is invariant under the group of isometries. Let $j=\left(j_{1}, \ldots, j_{d}\right) \in\{-1,1\}^{d}$, and let $g_{j}$ be the isometry mapping $p=\left(x_{1}, \ldots, x_{d}\right) \in E^{d}$ to $g_{j}(p)=\left(z_{1}, \ldots, z_{d}\right)$, where

$$
\left(\begin{array}{c}
z_{1} \\
\vdots \\
z_{d}
\end{array}\right)=\left(\begin{array}{cccc}
j_{1} & 0 & \cdots & 0 \\
0 & j_{2} & \cdots & 0 \\
\vdots & \vdots & \ddots & \vdots \\
0 & 0 & \cdots & j_{d}
\end{array}\right) \times\left(\begin{array}{c}
x_{1}-\frac{\gamma_{1}+1}{2} \\
\vdots \\
x_{d}-\frac{\gamma_{d}+1}{2}
\end{array}\right)+\left(\begin{array}{c}
\frac{\gamma_{1}+1}{2} \\
\vdots \\
\frac{\gamma_{d}+1}{2}
\end{array}\right)
$$


Since $K$ is centrally symmetric about $\left(\left(\gamma_{1}+1\right) / 2, \ldots,\left(\gamma_{d}+1\right) / 2\right)$, each transformation $g_{j}$ carries $K$ into itself. Given a point $q=\left(y_{1}, \ldots, y_{d}\right) \in E^{d} \backslash\{O\}$, let $\sigma(q)$ be the sign vector $\left(j_{1}, \ldots, j_{d}\right)$, where $j_{i}=1$ (resp. $j_{i}=-1$ ) if $y_{i} \geq 0$ (resp. $y_{i}<0$ ). To complete the proof, we will show that $g_{\sigma(q)}$ maps $S_{q}$ into $S_{\hat{q}}$, where $\hat{q} \in E_{+}^{d}$. The inverse transformation $g_{\sigma(q)}^{-1}$ maps a point $\left(x_{1}, \ldots, x_{d}\right)$ into $\left(z_{1}, \ldots, z_{d}\right)$, where

$$
z_{i}=j_{i} x_{i}+\left(1-j_{i}\right)\left(1+\gamma_{i}\right) / 2 \text {. }
$$

Consequently, the slab $S_{\hat{q}}$ is the set of points $\left(x_{1}, \ldots, x_{d}\right) \in E^{d}$ such that

$$
\left|\sum_{1 \leq i \leq d} j_{i} x_{i} y_{i}+\frac{1}{2} \sum_{1 \leq i \leq d}\left(1-j_{i}\right)\left(1+\gamma_{i}\right) y_{i}-\sum_{1 \leq i \leq d} y_{i}^{2}\right| \leq \alpha \sqrt{\sum_{1 \leq i \leq d} y_{i}^{2}} .
$$

We obtain an equivalent expression by noticing that

$$
\beta=\left(\sum_{1 \leq i \leq d}\left(1-j_{i}\right)\left(1+\gamma_{i}\right) y_{i}\right) / \sum_{1 \leq i \leq d} y_{i}^{2} \leq 0,
$$

and multiplying the previous inequality by $1-\beta / 2$. This shows that $\hat{q}=$ $\left(\hat{y}_{1}, \ldots, \hat{y}_{d}\right)$, where

$$
\hat{y}_{i}=(1-\beta / 2) j_{i} y_{i} \geq 0 .
$$

Noticing that $|\hat{q}|=(1-\beta / 2)|q|>0$, we can conclude that $\hat{q} \in E_{+}^{d}$.

Throughout this section the term "constant" refers to a quantity which may depend only on $d$, and not on $\alpha$ or any other parameter later defined. We will use $c$ as a generic symbol to denote a constant, avoiding subscripts whenever we can. Sometimes, however, we will have to resort to subscripts to be able to distinguish between different constants. The following result shows that if a slab is forced to contain a big hyperrectangle, then it cannot be moved around too much.

Lemma 4.4. For any dimension $d>1$, there exists a constant $c>0$ such that any hyperrectangle $K=\prod_{1 \leq i \leq d}\left[1, \gamma_{i}\right]$, where $\gamma_{i}>1$, satisfies the inequality $\lambda_{d}(K) \cdot \mu\left(H^{+}(K)\right)<c \alpha^{d+1}$.

Proof. We will assume throughout this proof that $q=\left(y_{1}, \ldots, y_{d}\right) \in E_{+}^{d}$ and $K \subseteq S_{q}$. To begin with, observe that $|q|$ can be neither too large nor too small. Indeed, since $(1,1, \ldots, 1) \in S_{q}$, we have

$$
\left.\left|\sum_{1 \leq i \leq d} y_{i}-\right| q\right|^{2}|\leq \alpha| q \mid \text {. }
$$

Since $x<1+x^{2} / 2$ for all $x$, this implies that

$$
|q|^{2}-\alpha|q| \leq \sum_{1 \leq i \leq d} y_{i}<d+|q|^{2} / 2
$$


from which we derive

$$
|q|<\alpha+\sqrt{\alpha^{2}+2 d} .
$$

Since $\alpha<1 / 12$ and $d>1$, it follows (conservatively) that $|q|<3 d$. Similarly, from (4.1) and the fact that $q \in E_{+}^{d}$, it follows that

$$
|q|^{2}+\alpha|q| \geq \sum_{1 \leq i \leq d} y_{i} \geq|q|,
$$

hence $|q| \geq 1-\alpha>1 / 2$. To summarize, we have shown that

$$
\frac{1}{2}<|q|<3 d \text {. }
$$

Let

$$
\Delta_{i}(K)=\left\{q=\left(y_{1}, \ldots, y_{d}\right) \in E_{+}^{d} \mid K \subseteq S_{q} \text { and } y_{i}>\frac{1}{2 \sqrt{d}}\right\} .
$$

From (4.2) it follows that $q$ belongs to at least one of the sets $\Delta_{i}(K) \quad(1 \leq i \leq$ d) ; therefore,

$$
\mu\left(H^{+}(K)\right) \leq \sum_{1 \leq i \leq d} M_{i}(K)
$$

where

$$
M_{i}(K)=\int_{\Delta_{i}(K)} \frac{d y_{1} \wedge \cdots \wedge d y_{d}}{|q|^{d-1}} .
$$

To estimate the value of $M_{i}(K)$, we set $i=1$ without loss of generality, in order to avoid overburdening the notation. Let us consider the following change of variables: given $q=\left(y_{1}, \ldots, y_{d}\right)$, let $u_{1}=\delta=|q|$, and for $i>1$, let $u_{i}=y_{i} / \delta$. Note that the transformation acts bijectively between

$$
\left\{\left(y_{1}, \ldots, y_{d}\right) \in E^{d} \backslash\{O\} \mid y_{1} \geq 0\right\}
$$

and

$$
\left\{\left(u_{1}, \ldots, u_{d}\right) \mid u_{1}>0 \text { and } \sum_{1<i \leq d} u_{i}^{2} \leq 1\right\} .
$$

To compute its Jacobian, $J_{u}$, we notice that

$$
\frac{\partial u_{i}}{\partial y_{j}}= \begin{cases}y_{j} / \delta, & \text { if } i=1 \\ 1 / \delta-y_{i}^{2} / \delta^{3}, & \text { if } i>1 \text { and } i=j \\ -y_{i} y_{j} / \delta^{3}, & \text { if } i>1 \text { and } i \neq j\end{cases}
$$

It follows that

$$
J_{u}=\left|\begin{array}{cccc}
y_{1} / \delta & y_{2} / \delta & \cdots & y_{d} / \delta \\
-y_{2} y_{1} / \delta^{3} & 1 / \delta-y_{2}^{2} / \delta^{3} & \cdots & -y_{2} y_{d} / \delta^{3} \\
\vdots & \vdots & \ddots & \vdots \\
-y_{d} y_{1} / \delta^{3} & -y_{d} y_{2} / \delta^{3} & \cdots & 1 / \delta-y_{d}^{2} / \delta^{3}
\end{array}\right| .
$$


We derive

$$
J_{u}=\frac{\left(\prod_{1 \leq i \leq d} y_{i}\right)^{2}}{y_{1} \delta^{3 d-2}} \times\left|\begin{array}{cccc}
+1 & +1 & \cdots & +1 \\
-1 & \left(\delta / y_{2}\right)^{2}-1 & \cdots & -1 \\
\vdots & \vdots & \ddots & \vdots \\
-1 & -1 & \cdots & \left(\delta / y_{d}\right)^{2}-1
\end{array}\right|
$$

The determinant above is made triangular by subtracting the first column from the others, which gives $J_{u}=y_{1} / \delta^{d}$. If $\Upsilon=\int_{q \in \Delta_{1}(K)} d u_{1} \wedge \cdots \wedge d u_{d}$, we immediately derive

$$
\Upsilon=\int_{\left(y_{1}, \ldots, y_{d}\right) \in \Delta_{1}(K)} \frac{y_{1}}{\left(y_{1}^{2}+\cdots+y_{d}^{2}\right)^{d / 2}} d y_{1} \wedge \cdots \wedge d y_{d}
$$

From (4.2) and the definition of $\Delta_{1}(K)$, we have

$$
\Upsilon \geq \frac{1}{6 d \sqrt{d}} M_{1}(K)
$$

If $q \in \Delta_{1}(K)$, then we have $\left.|\langle p, q\rangle-| q\right|^{2}|\leq \alpha| q \mid$, for both $p=(1, \ldots, 1)$ and $p=\left(\gamma_{1}, \ldots, \gamma_{d}\right)$. Since $q \in E_{+}^{d}$, this implies that

and from (4.2)

$$
-\alpha|q| \leq \sum_{1 \leq i \leq d} y_{i}-|q|^{2} \leq \sum_{1 \leq i \leq d} \gamma_{i} y_{i}-|q|^{2} \leq \alpha|q|,
$$

$$
\sum_{1 \leq i \leq d}\left(\gamma_{i}-1\right) y_{i} \leq 2 \alpha|q|<6 d \alpha
$$

Because $\gamma_{i}>1$, for each $i \quad(1 \leq i \leq d)$, we have

$$
0 \leq y_{i}<\frac{6 d \alpha}{\gamma_{i}-1}
$$

and for $i>1$,

$$
0 \leq u_{i} \leq \frac{2 \alpha}{\gamma_{i}-1}
$$

When $u_{2}, \ldots, u_{d}$ are fixed, $u_{1}$ always varies in an interval of length no greater than $2 \alpha$. It easily follows that

$$
\Upsilon \leq \frac{(2 \alpha)^{d}}{\prod_{1<i \leq d}\left(\gamma_{i}-1\right)}
$$

Note that the integration domain of $\Upsilon$ assumes that $y_{1}>1 /(2 \sqrt{d})$. From (4.5) we also have $y_{1}<6 d \alpha /\left(\gamma_{1}-1\right)$; therefore,

$$
\gamma_{1}-1<12 d \sqrt{d} \alpha \text {. }
$$

From (4.4) and (4.6) it then follows that

$$
M_{1}(K)<b \alpha^{d+1} / \lambda_{d}(K),
$$

for some constant $b$. The same inequality holds for any $M_{i}(K)$, so in view of (4.3) the proof is now complete.

Lemmas 4.3 and 4.4 give us a trade-off between the volume of a hyperrectangle and the measure of the slabs that contain it. We can use Lemma 4.2 to generalize this trade-off to any compact convex set. 
Lemma 4.5. For any dimension $d>1$, there exists a constant $c>0$ such that given any compact convex set $K$, we have $\lambda_{d}(K) \cdot \mu(H(K))<c \alpha^{d+1}$.

Proof. We can assume that $\lambda_{d}(K)>0$; otherwise, the lemma is obvious. Lemma 4.2 shows the existence of a parallelotope $\Pi \subseteq K$ such that

$$
\lambda_{d}(K) \leq d^{d} \lambda_{d}(\Pi) .
$$

Since $\Pi$ is not of measure zero, it is congruent to a hyperrectangle of the form $K^{\prime}=\prod_{1 \leq i \leq d}\left[1, \gamma_{i}\right]$, where $\gamma_{i}>1$. Obviously, the two sets $H(\Pi)$ and $H\left(K^{\prime}\right)$ have the same $\mu$-measure. (Note that the two sets might not be congruent, because $S_{q}$ is defined for $q \neq O$, and $E^{d} \backslash\{O\}$ is obviously not closed under the group of isometries. The difference, however, is simply a set of slabs whose bisecting hyperplanes pass through the origin, and thus has measure zero.) From Lemmas 4.3 and 4.4 , it follows that for some constant $c>0$,

$$
\lambda_{d}(\Pi) \cdot \mu(H(\Pi))<c 2^{d} \alpha^{d+1} .
$$

From (4.7) we derive

$$
\lambda_{d}(K) \cdot \mu(H(\Pi))<c(2 d)^{d} \alpha^{d+1} .
$$

Since $\Pi \subseteq K$ the proof is now complete.

We now turn to the special case where $K$ is of measure 0 . While Lemma 4.3 is still meaningful, Lemma 4.5 becomes trivial and must be modified a little. Recall that $D(K)$ denotes the diameter of the point-set $K$.

Lemma 4.6. For any dimension $d>1$, there exists a constant $c>0$ such that given any compact convex set $K$, we have $D(K) \cdot \mu(H(K))<c \alpha^{2}$.

Proof. We follow the proof of Lemma 4.4, assuming that $K$ is a hyperrectangle of the form $\prod_{1 \leq i \leq d}\left[1, \gamma_{i}\right]$, where $\gamma_{1}>1$ and $\gamma_{i}=1$, for $i>1$. It suffices to show that $\left(\gamma_{1}-1\right) \mu(H(K))<c \alpha^{2}$. From (4.5) we have $0 \leq y_{1}<6 d \alpha /\left(\gamma_{1}-1\right)$, and from (4.2) we derive that $0 \leq y_{j}<3 d$, for $1<j \leq d$. Consider the case of $M_{i}(K)$, for $i>1$. We have $0 \leq u_{1} \leq 2 \alpha /\left(\gamma_{1}-1\right), u_{i}=|q|$, and for $j>1$ and $j \neq i$, we have $0 \leq u_{j}<6 d$. This shows that

$$
\Upsilon \leq \frac{4 \alpha^{2}(6 d)^{d-2}}{\gamma_{1}-1}
$$

and from (4.4)

$$
M_{i}(K) \leq \frac{4 \sqrt{d}(6 d)^{d-1} \alpha^{2}}{\gamma_{1}-1}
$$

If we assume that $6 d \alpha /\left(\gamma_{1}-1\right)<1 /(2 \sqrt{d})$, then $\Delta_{1}(K)$ is empty, and hence $M_{1}(K)=0$. From (4.3) and (4.8) we find

$$
\mu\left(H^{+}(K)\right)<(6 d)^{d+2} \alpha^{2} /\left(\gamma_{1}-1\right) .
$$


Is this inequality still true if we relax the assumption on $\gamma_{1}$ ? If $6 d \alpha /\left(\gamma_{1}-1\right) \geq$ $1 /(2 \sqrt{d})$, then using the previous reasoning, we find $u_{1}=|q|$, and $0 \leq u_{j}<6 d$ $(1<j \leq d)$, from which it follows that

$$
M_{1}(K) \leq \sqrt{d}(2 \alpha)(6 d)^{d}<(6 d)^{d+2} \alpha^{2} /\left(\gamma_{1}-1\right) .
$$

From (4.9) we derive that

$$
\mu\left(H^{+}(K)\right)<2(6 d)^{d+2} \alpha^{2} /\left(\gamma_{1}-1\right),
$$

for all values of $\gamma_{1}>1$. Lemma 4.3 and the invariance of $\mu$ complete the proof.

\subsection{Approximating uniform point-set distributions.}

4.3.1. Introduction. We study the following discrepancy problem: Can we place $n$ points in $\mathscr{C}_{d}=[0,1]^{d}$ so that every subset of $k>d$ points has a convex hull of measure at least proportional to $k / n$ ? This is the kind of result we need in order to argue that big clusters occupy a lot of space. Let us consider the case $d=2$ and $k=3$ for a moment. This is known as Heilbronn's problem: What is the largest area, over all point-sets $P \subset \mathscr{C}_{2}$ of size $n$, of the smallest triangle with vertices in $P$ ?

This problem has a rich history. If we look at the one-dimensional case for inspiration, we might expect that in two dimensions the max-min area should be proportional to $1 / n$. However, it has been shown by Komlós, Szemerédi, and Pintz [12] that any set of $n$ points in $\mathscr{C}_{2}$ always contains a triangle of area less than $1 / n^{8 / 7-\varepsilon}$, for any $\varepsilon>0$. On the other hand, the same authors have shown [13] the existence of point-sets with all $\left(\begin{array}{l}n \\ 3\end{array}\right)$ triangles of area $\Omega\left((\log n) / n^{2}\right)$. See Moser [16] for a chronology of results on Heilbronn's problem.

At the other extreme-the case $k=\Omega(n)$-we have what we would expect: the max-min area of the convex hull of any subset of $k$ distinct points is $\Theta(k / n)$. Take the vertices of the largest regular $n$-gon inscribed in $\mathscr{C}_{2}$. A natural question is thus to determine the smallest function $k(n)$ for which the $\max -\min$ area is $\Omega(k(n) / n)$. More generally, let

$$
\Delta_{d}(n, k)=\max _{P \subseteq \mathscr{C}_{d}} \min _{\substack{S \subseteq P \\|P|=n}} \lambda_{d}(\kappa \mid \geq k
$$

where $d<k \leq n$; recall that $\kappa(S)$ denotes the convex hull of $S$. We know that $\Delta_{d}(n, n)=\Theta(n / n)$, but also that $\Delta_{2}(n, 3)=o(3 / n)$. We will use a probabilistic argument to prove that $\Delta_{d}(n, k)=\Theta(k / n)$, for any $k$ such that $\log n \leq k \leq n$.

4.3.2. On a generalization of Heilbronn's problem. We begin with some terminology. Let $\nu$ be a positive integer. We say that a finite set $P$ of points in $E^{d}$ is $\nu$-scattered if, for every subset $S \subseteq P$ of size $k \geq \nu$, we have $\lambda_{d}(\kappa(S))>\left(1 / 7^{7^{d}}\right) k /|P|$. As it turns out, a weaker version of this definition will allow us to sharpen our lower bounds for range searching in the case $d=2$. Given any positive real $\varepsilon$, the set $P$ is weakly $\varepsilon$-scattered if there exists a subset $\mathscr{Q}$ of $P$ such that

(i) $|\mathscr{Q}| \geq \varepsilon|P|$;

(ii) for every subset $S \subseteq \mathscr{Q}$ of size $k>d$, we have $\lambda_{d}(\kappa(S))>\varepsilon k /(10|P|)$. 
Let $R$ be a parallelotope in $E^{d}$ of nonzero measure; the $k$-faces of $R$ are called vertices if $k=0$, and edges if $k=1$. By edge-length, we refer to the Euclidean distance between the two vertices at the endpoints of an edge. There are at most $d$ distinct edge-lengths, $a_{1}, \ldots, a_{d}$ : the minimum value of $a_{i}$ is the width of $R$, while $\sqrt{a_{1}^{2}+\cdots+a_{d}^{2}}$ is its diameter. Let $\theta$ be a positive real. We define the $\theta$-pads of $R$ as a collection of $2^{d}$ parallelotopes parallel to $R$, of edgelength $\theta$, attached to each vertex of $R$. More precisely, let $g$ be an isometry carrying $R$ to the hyperrectangle $\prod_{1 \leq i \leq d}\left[0, a_{i}\right]\left(a_{i}>0\right)$. Each vertex $v$ of the hyperrectangle is of the form $v=\left(j_{1} a_{1}, \ldots, j_{d} a_{d}\right)$, where $\left(j_{1}, \ldots, j_{d}\right)$ is a bit-vector in $\{0,1\}^{d}$. We define the $\theta$-pad of the vertex $g^{-1}(v)$ of $R$ as the image under $g^{-1}$ of the hypercube of edge-length $\theta$ centered at the point

$$
\left(j_{1} a_{1}-(-1)^{j_{1}} \theta / 2, \ldots, j_{d} a_{d}-(-1)^{j_{d}} \theta / 2\right) \text {. }
$$

The notion of $\theta$-pads is useful for approximating the set of all convex subsets of $\mathscr{C}_{d}$ by a finite number of canonical polytopes. We begin by listing a few interesting properties of $\theta$-pads.

Lemma 4.7. Let $R$ be a parallelotope in $E^{d}$, and let $\theta$ be a positive real. If $S$ is a point-set which intersects each $\theta$-pad of $R$, then the convex hull of $S$ contains $R$.

Proof. Without loss of generality, we may assume that $R$ is a hyperrectangle of the form $\prod_{1 \leq i \leq d}\left[0, a_{i}\right]$. Let $s_{1}, \ldots, s_{2^{d}}$ be representative points of $S$ in each $\theta$-pad of $R$, with $s_{1}$ the point in the $\theta$-pad of $O$. Any (closed) halfspace that contains $O$ also contains at least one $s_{i}$. To see this, consider the hyperplanes

$$
P_{q}=\left\{\left.p \in E^{d}|\langle p, q\rangle=| q\right|^{2}\right\},
$$

and notice that for each sign assignment of the coordinates of $q$ there is at least one desirable $s_{i}$. This shows that $O \in \kappa\left(\left\{s_{1}, \ldots, s_{2^{d}}\right\}\right)$. By symmetry, the same is true of all the other vertices; therefore, their convex hull, $R$, lies inside $\kappa\left(\left\{s_{1}, \ldots, s_{2^{d}}\right\}\right)$.

The next lemma provides a polynomial-size approximation of the set of all convex subsets of $\mathscr{C}_{d}$ of measure $\rho$. This enables us to use discrete probabilistic techniques to study certain uniformity criteria for point-sets (Lemma 4.9).

Lemma 4.8. For any $d>1$ and any real $\rho(0<\rho \leq 1)$, there exists $a$ collection $\mathfrak{F}$ of convex sets such that (i) $|\mathfrak{F}|<5^{5^{d}} / \rho^{d 2^{d}}$, (ii) for each $C \in \mathfrak{F}$, we have $C \subseteq \mathscr{C}_{d}$ and $\lambda_{d}(C)<5^{5^{d}} \rho$, (iii) given any convex set $K$ in $\mathscr{C}_{d}$ of measure $\rho$, there exists some $C \in \mathfrak{F}$ which contains $K$.

Proof. Let $\beta=\rho / d^{(3 d+1) / 2}$, and let $\mathscr{G}$ be the grid of points

$$
\mathscr{G}=\{ \pm i \beta \mid i \geq 0\}^{d} \cap[-2 d, 2 d]^{d} \text {. }
$$

We define $\mathfrak{F}$ as follows:

$$
\mathfrak{F}=\left\{\kappa(S) \cap \mathscr{C}_{d} \mid S \subseteq \mathscr{G} \text { and }|S|=2^{d} \text { and } \rho \leq \lambda_{d}(\kappa(S))<3^{d} d ! \rho\right\} .
$$


We have $|\mathscr{G}|=(2\lfloor 2 d / \beta\rfloor+1)^{d}$, which implies that $|\mathscr{G}|<5^{d} d^{3 d(d+1) / 2} / \rho^{d}$, since $\rho \leq 1<d$, and hence (i). Note that (ii) follows directly from the definition of $\mathfrak{F}$, so let us turn our attention to (iii).

Let $K$ be a convex set in $\mathscr{C}_{d}$ of measure $\rho$, and let $R$ (resp. $r$ ) be the circumscribed (resp. inscribed) parallelotope of least (resp. greatest) measure. From Lemma 4.2, it follows that

$$
\lambda_{d}(R) / d ! \leq \rho \leq d^{d} \lambda_{d}(r) .
$$

Let $\Psi$ be the set of $(\beta \sqrt{d})$-pads of $R$. It is not difficult to show that each pad $\psi$ of $\Psi$ contains a grid point. Let $\xi$ be the center of $\psi$, and let $\mathscr{G}^{*}$ be the infinite grid $\{ \pm i \beta \mid i \geq 0\}^{d}$. There is a point $\gamma \in \mathscr{G}^{*}$ within a distance $\sqrt{d} \beta / 2$ of $\xi$; therefore, $\gamma$ lies within the pad $\psi$. We must now show that $\gamma$ is actually a point of $\mathscr{G}$. By construction, no edge-length of $R$ can exceed the diameter of $K$. Since $K$ lies in $\mathscr{C}_{d}$, its diameter is at most $\sqrt{d}$. This implies that the diameter of $R$ is at most $d$. Because the diameter of $\psi$ is equal to $d \beta$, it follows that the distance from $\gamma$ to $O$ is at most $d+\sqrt{d}+d \beta$, which is less than $2 d$, since $\rho \leq 1$ and $d \geq 2$. This proves that $\gamma$ belongs to $[-2 d, 2 d]^{d}$, and therefore, is a point of $\mathscr{G}$.

We thus have established the existence of a set $S$ of $2^{d}$ points in $\mathscr{G}$, each of which lies in a distinct $(\beta \sqrt{d})$-pad of $R$. Since $r$ lies inside $\mathscr{C}_{d}$, its maximum edge-length is at most $\sqrt{d}$; therefore, the width of $r$, and hence the width of $R$, is at least $\lambda_{d}(r) / d^{(d-1) / 2}$. From (4.10) it then follows that the width of $R$ is at least $\rho \sqrt{d} / d^{3 d / 2}$. Let $l_{1}, \ldots, l_{d}$ be the edge-lengths of $R$. We have

$$
l_{i} \geq \rho \sqrt{d} / d^{3 d / 2}>\beta \sqrt{d}
$$

therefore,

$$
\lambda_{d}(\kappa(S)) \leq \prod_{1 \leq i \leq d}\left(l_{i}+2 \beta \sqrt{d}\right)<3^{d} \lambda_{d}(R) .
$$

From Lemma 4.7 and (4.10) we derive

$$
\rho \leq \lambda_{d}(\kappa(S))<3^{d} d ! \rho .
$$

This proves that $\kappa(S) \cap \mathscr{C}_{d} \in \mathfrak{F}$ and therefore $K \subseteq \kappa(S) \cap \mathscr{C}_{d}$.

Lemma 4.9. For any $d>1$ and $n$ sufficiently large, a random set of $n$ points in $\mathscr{C}_{d}$ is $(\log n)$-scattered with probability greater than $1-1 / n$.

Proof. Let $c=1 /\left(2^{4^{d}} b\right)$, where $b=5^{5^{d}}$ is the constant used in Lemma 4.8. Note that $c>1 / 7^{7^{d}}$. Throughout the proof, we will use the notation of Lemma 4.8 , with the value of $\rho$ set to $3 c(\log n) / n$. (Note that this assignment is valid, since for $n>1$ we have $0<\rho<1$.) We shall also assume that $n$ is larger than some appropriate constant. Let $P$ be a random set of $n$ points in $\mathscr{C}_{d}$, and let $\pi$ be the probability that there exists a convex set $K \subseteq \mathscr{C}_{d}$ such that $k=|K \cap P| \geq \log n$ and $\lambda_{d}(K) \leq c k / n$. We can assume that the $n$ points of $P$ are distinct since this happens with probability 1 . It is then possible to partition $K$ into convex sets, each containing between $\log n$ and $2 \log n+1$ 
points. To do so, choose a line $L$ which is not normal to any of the hyperplanes passing through a pair of points in $K \cap P$, and sort the projection of the points of $K \cap P$ onto $L$. Since there are no identical elements in the resulting list, we can partition it into sublists of size $\lceil\log n\rceil$ (except for the last one, whose size falls between $\lceil\log n\rceil$ and $2\lceil\log n\rceil-1)$. For each pair of adjacent sublists, find a point on $L$ separating them and cut $K$ by the hyperplane normal to $L$ passing through the point. Of the pieces of $K$ thus created, let $K^{*}$ be the one of smallest measure. We have

$$
\lambda_{d}\left(K^{*}\right) \leq 3 c \frac{\log n}{n}=\rho
$$

therefore, we can always enclose $K^{*}$ inside a convex set $\subseteq \mathscr{C}_{d}$ of measure $\rho$. From Lemma 4.8, it follows that the collection $\mathfrak{F}$ contains at least one set $C$ which encloses $K^{*}$, where

$$
\lambda_{d}(C)<3 b c \frac{\log n}{n} .
$$

Clearly, the set $C$ contains at least $\log n$ points of $P$; therefore,

$$
\pi<\sum_{C \in \mathfrak{F}} \sum_{j \geq \log n}\left(\begin{array}{l}
n \\
j
\end{array}\right) \lambda_{d}^{j}(C)\left(1-\lambda_{d}(C)\right)^{n-j} .
$$

From (4.11) we have $n \lambda_{d}(C)<\log n$; therefore, we can use the Chernoff bound [8] to approximate the tail of the binomial distribution. This yields

$$
\pi<b\left(\frac{n}{3 c \log n}\right)^{d 2^{d}} \times\left(\frac{n\left(1-\lambda_{d}(C)\right)}{n-\log n}\right)^{n-\log n} \times\left(\frac{n \lambda_{d}(C)}{\log n}\right)^{\log n} .
$$

Using Taylor's expansion, we have

$$
\ln (1-(\log n) / n)>-(\log n) / n-(\log n)^{2} / n^{2},
$$

for $n$ large enough; therefore,

$$
n^{2}(n-\log n)^{n-\log n}>n^{n-\log n} .
$$

On the other hand, it follows from (4.11) that

$$
\left(n \lambda_{d}(C) / \log n\right)^{\log n}<1 / n^{4^{d}-2} .
$$

Putting (4.12)-(4.14) together, we find the desired (conservative) upper bound $\pi<1 / n$.

As an immediate corollary, we obtain this new result on the generalization of Heilbronn's problem. Whether $\log n$ can be replaced by anything smaller (asymptotically) is an intriguing open problem.

Theorem 4.10. The function $\Delta_{d}(n, k)$ is in $\Theta(k / n)$, for any $k$ such that $\log n \leq$ $k \leq n$.

Proof. Because of Lemma 4.9 it suffices to show that $\Delta_{d}(n, k)=O(k / n)$. Given any set $P$ of $n$ points in $\mathscr{C}_{d}$, partition $\mathscr{C}_{d}$ into convex sets, each containing between $k$ and $2 k+1$ points (using, for example, the method given 
in Lemma 4.9). Now, consider the convex hull of the set of smallest measure. This set contains at least $\lceil k\rceil$ points and its measure is $O(k / n)$.

Simplex range searching in 2-space requires a special treatment. The idea is to adopt the weak version of scattering, which then allows us to "strengthen" Lemma 4.9 in two dimensions.

Lemma 4.11. There exists a positive real $\varepsilon_{0}<1$ such that for any $\varepsilon\left(0<\varepsilon<\varepsilon_{0}\right)$ and any $n>2$, a random set of $n$ points in $\mathscr{C}_{1}$ is weakly $\varepsilon$-scattered with probability greater than $1-\varepsilon$.

Proof. Consider the inequalities

$$
1-\varepsilon / 2<\left(1-\theta^{2}\right) e^{-\theta}<1-\theta / 2<1-2 \varepsilon / 9 .
$$

We claim that there exists some real $\varepsilon_{0} \quad\left(0<\varepsilon_{0}<1 / 2\right)$ such that for any $\varepsilon$, where $0<\varepsilon<\varepsilon_{0}$, there exists $\theta$ which satisfies (4.15). To see this it suffices to notice that if $1-\varepsilon / 2=\left(1-\theta^{2}\right) e^{-\theta}$ then $|\theta-\varepsilon / 2|=O\left(\varepsilon^{2}\right)$, and

$$
1-\theta / 2-\left(1-\theta^{2}\right) e^{-\theta}=\theta / 2+O\left(\theta^{2}\right)=\varepsilon / 4 \pm O\left(\varepsilon^{2}\right) .
$$

Let $P$ be a random set of $n>2$ reals in $[0,1]$. We say that $x \in P$ is isolated if there is no other element of $P$ in $[x-\theta /(2 n), x+\theta /(2 n)]$. Let $\nu$ be the expected number of isolated points, and let $\pi$ be the probability that at least $\varepsilon n$ points of $P$ are isolated. We have

$$
\nu \leq(1-\pi) \varepsilon n+\pi n \text {. }
$$

On the other hand, we have $\nu \geq n(1-\theta / n)^{n-1}$. Since $0<\theta<\varepsilon<1<n$, we have (Abramowitz and Stegun [1, p. 68])

$$
\nu>n(1-\theta / n)^{n}=n e^{n \ln (1-\theta / n)}>n e^{-\theta /(1-\theta / n)} .
$$

Using the inequalities $e^{x} \geq 1+x$ and $n>2 \theta$, we derive

$$
\nu>n e^{-\theta(1+2 \theta / n)} \geq n e^{-\theta}\left(1-2 \theta^{2} / n\right) .
$$

Using (4.15) and (4.16) and the inequalities $n>2$ and $\varepsilon<1 / 2$, we have

$$
\pi \geq \frac{\nu / n-\varepsilon}{1-\varepsilon}>\frac{\left(1-\theta^{2}\right) e^{-\theta}-\varepsilon}{1-\varepsilon}>\frac{1-3 \varepsilon / 2}{1-\varepsilon}>1-\varepsilon .
$$

On the other hand, the convex hull of any $k>1$ isolated points is an interval of length at least $\frac{1}{2}(k-1) \theta / n$, which from (4.15) exceeds $\varepsilon k /(10 n)$.

4.4. The lower bounds on simplex range searching. We are now in a position to attack our original problem. Let us recall our assumptions. The dimension $d$ is at least 2, and the parameter $\alpha$ is a positive real less than $\frac{1}{12}$. Let $m$ and $n$ be two positive integers, and let $\Gamma$ be a function mapping any set $P$ of $n$ points in $\mathscr{C}_{d}$ to a storage scheme for $P$ of size $m>0$. When $P$ and $\Gamma(P)$ are understood, we write $t$ to denote the worst case time complexity $\max _{q \in t} t(P, \Gamma(P), q)$. It will be important to keep in mind later on that $t$ is actually a parameter depending on $P$ and $\Gamma$. Ironically, the higher-dimensional case $(d \geq 3)$ is easier to handle, so this is where we begin our investigation. 
4.4.1. Range searching in $d$-space. Let $B_{d}(\rho)$ be the closed $d$-dimensional ball of radius $\rho$ centered at $\left(\frac{1}{2}, \ldots, \frac{1}{2}\right)$. We define the query space $\mathscr{Q}$ to be the set of slabs $\left\{S_{q} \mid q \in B_{d}\left(\frac{1}{4}\right)\right\}$. We begin our investigation with a technical lemma saying that every query grabs a reasonable chunk of the unit $d$-cube: neither too big nor too small.

Lemma 4.12. For any $d>1$ there exists a constant $0<c_{1}<1$ such that for any $S_{q} \in \mathscr{Q}$, we have $c_{1} \alpha<\lambda_{d}\left(S_{q} \cap \mathscr{C}_{d}\right)<\alpha / c_{1}$.

Proof. Because $S_{q} \cap B_{d}\left(\frac{1}{2}\right) \subseteq S_{q} \cap \mathscr{C}_{d}$ we have $\lambda_{d}\left(S_{q} \cap \mathscr{C}_{d}\right)>2 \alpha \lambda_{d-1}\left(B^{\prime}\right)$, where $B^{\prime}$ is the intersection of $B_{d}\left(\frac{1}{2}\right)$ with a hyperplane at distance $\frac{1}{4}+\alpha$ from the center of $B_{d}\left(\frac{1}{2}\right)$. This implies that $B^{\prime}$ is a ball in $E_{d-1}$ of radius $r=\sqrt{\frac{1}{4}-\left(\frac{1}{4}+\alpha\right)^{2}}$. Its $(d-1)$-dimensional measure is therefore (Santaló [17])

$$
\lambda_{d-1}\left(B^{\prime}\right)=\frac{2 \pi^{(d-1) / 2} r^{d-1}}{(d-1) \Gamma((d-1) / 2)},
$$

where $\Gamma$ is the gamma function. Using simple approximations we easily verify that, since $\alpha<\frac{1}{12}$, we have $\lambda_{d}\left(S_{q} \cap \mathscr{C}_{d}\right)>c \alpha$, where

$$
c=\frac{\pi^{(d-1) / 2}}{(d-1) 3^{d} \Gamma((d-1) / 2)} .
$$

Conversely, the diameter of $\mathscr{C}_{d}$ is equal to $\sqrt{d}$; therefore, $\lambda_{d}\left(S_{q} \cap \mathscr{C}_{d}\right)<$ $2 \alpha \lambda_{d-1}\left(B^{*}\right)$, where $B^{*}$ is a $(d-1)$-dimensional ball of radius $\sqrt{d} / 2$. We derive

$$
\lambda_{d}\left(S_{q} \cap \mathscr{C}_{d}\right)<\frac{\alpha \pi^{(d-1) / 2} d^{(d-1) / 2}}{(d-1) 2^{d-3} \Gamma((d-1) / 2)} .
$$

Given a set $P$ of $n$ points in $\mathscr{C}_{d}$, we say that a slab $S_{q}$ is heavy if $S_{q} \in \mathscr{Q}$ and $\left|S_{p} \cap P\right|>c_{1} \alpha n / 2$. We focus on heavy query slabs because they are both well positioned and reasonably filled with points of $P$. Our next result says that this focusing is not too restrictive when dealing with a random point-set $P$. A random query of $\mathscr{Q}$ is heavy with high probability.

Lemma 4.13. For any $d>1$ there exists a constant $c>0$ such that for any fixed real $\varepsilon \quad(0<\varepsilon<1)$ and a random set of $n$ points in $\mathscr{C}_{d}$, the measure of the set of heavy slabs exceeds $(1-c /(\alpha \varepsilon n)) \mu(\mathscr{Q})$ with probability greater than $1-\varepsilon$.

Proof. Let $P$ be a random set of $n$ points in $\mathscr{C}_{d}$, and let $S_{q}$ be a slab of $\mathscr{Q}$. Put $\chi=\left|S_{q} \cap P\right|$ and $\sigma=S_{q} \cap \mathscr{C}_{d}$. The mean and variance of $\chi$ are, respectively, $n \lambda_{d}(\sigma)$ and $n \lambda_{d}(\sigma)\left(1-\lambda_{d}(\sigma)\right)$. Let $\pi(q)$ be the probability that $S_{q}$ is heavy with respect to a random $P$. Combining Lemma 4.12 and Chebyshev's inequality, we find

$$
1-\pi(q) \leq \operatorname{Prob}\left(\left|\chi-n \lambda_{d}(\sigma)\right| \geq c_{1} \alpha n / 2\right) \leq \frac{4 n \lambda_{d}(\sigma)\left(1-\lambda_{d}(\sigma)\right)}{c_{1}^{2} \alpha^{2} n^{2}}
$$


Again from Lemma 4.12 it follows that

$$
\pi(q)>1-\frac{4}{c_{1}^{3} \alpha n} .
$$

By Fubini's theorem, the expected value $E$ of the measure of the set of heavy slabs is equal to $\int_{S_{q} \in \mathscr{Q}} \pi(q) d S_{q}$, which from (4.17) gives

$$
E>\left(1-\frac{4}{c_{1}^{3} \alpha n}\right) \mu(\mathscr{Q}) \text {. }
$$

On the other hand, we have

$$
E \leq(1-p)\left(1-\frac{5}{c_{1}^{3} \alpha \varepsilon n}\right) \mu(\mathscr{Q})+p \mu(\mathscr{Q}),
$$

where $p$ is the probability that the measure of the set of heavy slabs is at least $\left(1-5 /\left(c_{1}^{3} \alpha \varepsilon n\right)\right) \mu(\mathscr{Q})$. This inequality, combined with (4.18), shows that $p>1-\varepsilon$, which completes the proof.

Let $p_{1}, \ldots, p_{n}$ be the points of $P$. Recall that each generator $g$ of $\Gamma(P)$ is a linear form $\sum_{1 \leq i \leq n} \alpha_{i} s_{i}$ and that its cluster is the set $\left\{p_{i} \mid \alpha_{i} \neq 0\right\}$. By abuse of notation we will refer to the clusters of $\Gamma(P)$. From the equivalence result of Lemma 2.1 we know that, for each $S_{q} \in \mathscr{Q}$, the set $S_{q} \cap P$ can be expressed as the union of at most $t$ clusters. A heavy query contains $\Omega(\alpha n)$ points of $P$. To be answered in time $t$ therefore requires the use of clusters of size $\Omega(\alpha n / t)$. Just as we chose to focus on heavy queries, we will restrict our analysis to those "fat" clusters. Specifically, we say that a cluster is fat if it contains at least $\frac{1}{4} c_{1} \alpha n / t$ points. For any $S_{q} \in \mathscr{Q}$, let $\nu(q)$ be equal to the number of points in $S_{q} \cap P$ which belong to at least one fat cluster lying entirely within $S_{q}$. (Note that these clusters may not necessarily be used in answering the query $S_{q}$.) Our next result says that with a random point-set $P$ the average value of $\nu(q)$ (over all $S_{q} \in \mathscr{Q}$ ) is $\Omega(\alpha n)$.

Lemma 4.14. For any $d>1$ there exists a constant $c>0$ such that for any fixed real $\varepsilon(0<\varepsilon<1)$ and a random set of $n$ points in $\mathscr{C}_{d}$, the inequality

$$
\int_{\mathscr{Q}} \nu(q) d S_{q}>(\alpha n / c-c / \varepsilon) \mu(\mathscr{Q})
$$

holds true with probability greater than $1-\varepsilon$.

Proof. Given a random set $P$ and $S_{q} \in \mathscr{Q}$, let $C_{1}, \ldots, C_{u}$ be a set of clusters such that $u \leq t$ and $S_{q} \cap P=\cup_{1 \leq i \leq u} C_{i}$. By the pigeonhole principle, the number of points of $S_{q} \cap P$ that belong to at least one cluster $C_{i}$ such that $\left|C_{i}\right| \geq\left|S_{q} \cap P\right| /(2 u)$ exceeds $\left|S_{q} \cap P\right| / 2$. Suppose that $S_{q}$ is heavy. Then because $u \leq t$, we have $\nu(q)>c_{1} \alpha n / 4$. From Lemma 4.13 it follows that with probability greater than $1-\varepsilon$ we have

$$
\begin{aligned}
\int_{\mathscr{Q}} \nu(q) d S_{q} & >\frac{1}{4} c_{1} \alpha n\left(1-\frac{c}{\alpha \varepsilon n}\right) \mu(\mathscr{Q}) \\
& >\left(\alpha n /\left(4 / c_{1}+c_{1} c / 4\right)-\left(4 / c_{1}+c_{1} c / 4\right) / \varepsilon\right) \mu(\mathscr{Q}),
\end{aligned}
$$

where $c$ is the constant of Lemma 4.13. 
Suppose now that $P$ is $(\log n)$-scattered, with $n>2^{d}$, and let $S$ be a subset of $P$ of size at least $\log n$. By definition, we have $\lambda_{d}(\kappa(S))>\left(1 / 7^{7^{d}}\right)|S| / n$. From Lemma 4.5 we derive that for some constant $a_{1}>0$,

$$
|S| \cdot \mu(H(\kappa(S)))<a_{1} \alpha^{d+1} n \text {. }
$$

Put

$$
\alpha=\left(\frac{\mu(\mathscr{Q})}{3 a_{1} c m}\right)^{1 / d},
$$

where $c$ is the constant of Lemma 4.14, and suppose that we have the following relationship between the storage $m$ and the query time $t$ :

$$
m t^{d}<\frac{\left(c_{1} n\right)^{d} \mu(\mathscr{Q})}{a_{1} c(8 \log n)^{d}} .
$$

Observe that since $m \geq n$ the condition $\alpha<1 / 12$ is satisfied for any $n$ large enough. Let $C_{1}, \ldots, C_{\beta}$ be the fat clusters of $\Gamma(P)$. From (4.20) we find that any fat cluster contains more than $\log n$ points. Pursuing the basic approach behind the Core Lemma, we turn our attention to the key inequality

$$
\int_{\mathscr{Q}} \nu(q) d S_{q} \leq \sum_{1 \leq i \leq \beta}\left|C_{i}\right| \mu\left(H\left(\kappa\left(C_{i}\right)\right)\right) .
$$

From (4.19) it follows that

$$
\int_{\mathscr{Q}} \nu(q) d S_{q}<a_{1} \alpha^{d+1} \beta n
$$

Assume that

$$
m<\frac{(\varepsilon n)^{d} \mu(\mathscr{Q})}{a_{1}(4 c)^{2 d+1}} .
$$

Then Lemmas 4.9 and 4.14 imply that for any $n$ large enough and any $\varepsilon$ $(0<\varepsilon<1)$ a random set $P$ satisfies

$$
\frac{\alpha n}{2 c} \mu(\mathscr{Q})<a_{1} \alpha^{d+1} m n
$$

with probability greater than $1-\varepsilon-1 / n$. But this leads to a contradiction, so (4.20) or (4.21) must be false. Since $\mu(\mathscr{Q})$ is larger than some positive constant (independent of $\varepsilon$ ), we immediately derive the following result.

Lemma 4.15. For any $d>1$ and any $\varepsilon \quad(0<\varepsilon<1)$ there exists a constant $c>0$ such that for any $n>1 / c$, a random set of $n$ points in $\mathscr{C}_{d}$ satisfies $m t^{d}>c(n / \log n)^{d}$ with probability greater than $1-\varepsilon$.

4.4.2. Sharper bounds for 2-space. The two-dimensional version of Lemma 4.15 can be improved by using weak scattering and Lemma 4.11. The proof is slightly more technical, although the basic approach remains the same. We use the notation of the previous section. A parallelotope is now simply called a rectangle. Given a slab $S_{q}$, we define $R_{q}$ as the largest rectangle $S_{q} \cap \mathscr{C}_{2}$ 
with two sides collinear with the bounding lines of $S_{q}$. Since $\alpha<\frac{1}{12}, R_{q}$ is well defined and unique. As before, the query space $\mathscr{Q}$ is the set of slabs $\left\{S_{q} \mid q \in B_{2}\left(\frac{1}{4}\right)\right\}$. The proof of the following result is almost identical to that of Lemma 4.12, so we omit it.

Lemma 4.16. There exists a constant $0<c_{2}<1$ such that for any $S_{q} \in \mathscr{Q}$ we have $c_{2} \alpha<\lambda_{2}\left(R_{q}\right)<\alpha / c_{2}$.

We must strengthen the concept of heaviness by bringing into play the notion of weak scattering. Given a set $P$ of $n$ points in $\mathscr{C}_{2}$, we now say that a slab $S_{q}$ is $\varepsilon$-favorable if

(i) $S_{q} \in \mathscr{Q}$,

(ii) $c_{2} \alpha n / 2<\left|R_{q} \cap P\right|<2 \alpha n / c_{2}$, and

(iii) the orthogonal projection of the points of $R_{q} \cap P$ on either bounding line of $S_{q}$ is weakly $\left(c_{2} \varepsilon^{2} / 2\right)$-scattered in $E^{1}$.

We now have the analog of Lemma 4.13, saying that if we have a random point-set $P$, then a random query is $\varepsilon$-favorable with high probability.

Lemma 4.17. There exist two positive constants $c$ and $\varepsilon_{1}$ such that for any real $\varepsilon\left(0<\varepsilon<\varepsilon_{1}\right)$ and a random set of $n$ points in $\mathscr{C}_{2}$, where $\alpha n>c$, the measure of the set of $\varepsilon$-favorable slabs exceeds $(1-\varepsilon-c /(\alpha \varepsilon n)) \mu(\mathscr{Q})$ with probability greater than $1-\varepsilon$.

Proof. Let $\pi(q)$ be the probability that $S_{q}$ is $\varepsilon$-favorable, assuming that $S_{q} \in$ Q. We have $\pi(q)=\pi_{1}(q) \pi_{2}(q)$, where $\pi_{1}(q)$ is the probability that $c_{2} \alpha n / 2<$ $\left|R_{q} \cap P\right|<2 \alpha n / c_{2}$ and $\pi_{2}(q)$ is the conditional probability that the points of $R_{q} \cap P$ projected onto a bounding line of $S_{q}$ are weakly $\left(c_{2} \varepsilon^{2} / 2\right)$-scattered, given that $c_{2} \alpha n / 2<\left|R_{q} \cap P\right|<2 \alpha n / c_{2}$. Using Chebyshev's inequality we derive

$$
\pi_{1}(q) \geq 1-\frac{4}{c_{2}^{3} \alpha n} .
$$

On the other hand, since the point distribution is uniform in $\mathscr{C}_{2}$, given a fixed subset $S$ of $P \cap R_{q}$, the projection of $S$ onto a bounding line of $S_{q}$ is uniformly distributed along the corresponding side $s$ of $R_{q}$. From Lemma 4.16, the length of $s$ is at least $c_{2} / 2<1$, so it follows from Lemma 4.11 that if $\left|R_{q} \cap P\right|>$ $c_{2} \alpha n / 2$, then for $\varepsilon<\varepsilon_{1} \quad\left(\varepsilon_{1}>0\right)$ and $\alpha n \geq 4 / c_{2}$, the projection of $R_{q} \cap P$ is weakly $\left(c_{2} \varepsilon^{2} / 2\right)$-scattered with probability greater than $1-\varepsilon^{2}$. From (4.22) we then derive that the expected value $\Phi$ of the measure of the set of $\varepsilon$-favorable slabs satisfies

$$
\Phi \geq\left(1-\varepsilon^{2}\right)\left(1-\frac{4}{c_{2}^{3} \alpha n}\right) \mu(Q)
$$

But we also have

$$
\Phi \leq(1-p)\left(1-\varepsilon-\frac{5}{c_{2}^{3} \alpha \varepsilon n}\right) \mu(Q)+p \mu(Q),
$$


where $p$ is the probability that the measure of the set of $\varepsilon$-favorable slabs is at least $\left(1-\varepsilon-5 /\left(c_{2}^{3} \alpha \varepsilon n\right)\right) \mu(\mathscr{Q})$. Combining this inequality with (4.23), the lemma follows readily.

Let $P$ be a set of $n$ points in $\mathscr{C}_{2}$, and let $S_{q}$ be $\varepsilon$-favorable with respect to $P$. Then $S_{q}$ contains a subset $Q \subseteq P$ of size $\geq c_{2} \varepsilon^{2}\left|R_{q} \cap P\right| / 2$, with the following properties. Let $Q^{\prime}$ be the orthogonal projection of $Q$ onto a bounding line of $S_{q}$. Then for every subset $S \subseteq Q^{\prime}$ of at least two points, we have

$$
\lambda_{1}(\kappa(S))>\frac{c_{2} \varepsilon^{2}|S|}{20\left|R_{q} \cap P\right|} .
$$

Since $\left|R_{q} \cap P\right|<2 \alpha n / c_{2}$, this implies that given any subset $C$ of $Q$ of at least two points, the diameter of $C$ satisfies

$$
D(C)>\frac{c_{2}^{2} \varepsilon^{2}|C|}{40 \alpha n} .
$$

The subset $Q$ is called the prime subset of $S_{q}$. Since $S_{q}$ is $\varepsilon$-favorable, we have

$$
|Q| \geq c_{2}^{2} \varepsilon^{2} \alpha n / 4 .
$$

Although $Q$ is not necessarily unique we can always use a canonical ordering to make the prime subset unambiguously defined.

Next, we replace $\nu(q)$ by the function $\xi(\varepsilon, q)$, defined as follows. If $S_{q}$ is $\varepsilon$-favorable, then $\xi(\varepsilon, q)$ is the number of points in the prime subset of $S_{q}$ which share a cluster with at least another point. More precisely, let $\mathscr{F}$ be the set of clusters which lie entirely within $S_{q}$ and contain at least two points in the prime subset of $S_{q}$. Then

$$
\xi(\varepsilon, q)=\mid \bigcup_{C \in \mathscr{F}}\left(C \cap \text { prime subset of } S_{q}\right) \mid .
$$

If $S_{q}$ is not $\varepsilon$-favorable then $\xi(\varepsilon, q)=0$.

Lemma 4.18. There exist two positive constants $c$ and $\varepsilon_{1}$ such that for any real $\varepsilon\left(0<\varepsilon<\varepsilon_{1}\right)$ and a random set of $n$ points in $\mathscr{C}_{2}$ with $\alpha n / t>1 /\left(c \varepsilon^{2}\right)$ the inequality

$$
\int_{\mathscr{Q}} \xi(\varepsilon, q) d S_{q}>c \varepsilon^{2} \alpha n\left(1-\varepsilon-\frac{1}{c^{2} \alpha \varepsilon n}\right) \mu(\mathscr{Q})
$$

holds true with probability greater than $1-\varepsilon$.

Proof. Let $S_{q}$ be an $\varepsilon$-favorable slab, let $Q$ be its prime subset, and let $C_{1}, \ldots$, $C_{u}$ be a set of clusters such that $u \leq t$ and $S_{q} \cap P=\bigcup_{1 \leq i \leq u} C_{i}$. All but at most $u$ points of $Q$ belong to clusters $C_{i}$ each of which contains at least two points of $Q$. From (4.25) we derive that for $\alpha n / t>8 /\left(c_{2}^{2} \varepsilon^{2}\right)$,

$$
\xi(\varepsilon, q) \geq c_{2}^{2} \varepsilon^{2} \alpha n / 4-t>c_{2}^{2} \varepsilon^{2} \alpha n / 8 .
$$


Consequently, Lemma 4.17 shows that with probability greater than $1-\varepsilon$ a random. set $P$ satisfies

$$
\int_{\mathscr{Q}} \xi(\varepsilon, q) d S_{q}>\frac{1}{8} c_{2}^{2} \varepsilon^{2} \alpha n\left(1-\varepsilon-\frac{c}{\alpha \varepsilon n}\right) \mu(\mathscr{Q}),
$$

provided that $\alpha n>c$ and $\alpha n / t>8 /\left(c_{2}^{2} \varepsilon^{2}\right)$. Since $t \geq 1$, the lemma follows directly.

Let $C_{1}, \ldots, C_{m}$ be the clusters of $\Gamma(P)$, and for each $i \quad(1 \leq i \leq m)$, let $\eta_{i}$ be the maximum number of points in $C_{i}$ to be in the prime subset of the same $\varepsilon$-favorable slab $S_{q}$. If this number is strictly less than 2 , then we set $\eta_{i}=0$. Clearly,

$$
\int_{\mathscr{Q}} \xi(\varepsilon, q) d S_{q} \leq \sum_{1 \leq i \leq m} \eta_{i} \mu\left(H\left(\kappa\left(C_{i}\right)\right)\right)
$$

Using (4.24) for each $i$ such that $\eta_{i}>0$, as well as Lemma 4.6, we derive

$$
\int_{\mathscr{Q}} \xi(\varepsilon, q) d S_{q}<\left(\frac{40 \alpha n}{c_{2}^{2} \varepsilon^{2}}\right) \sum_{1 \leq i \leq m} D\left(\kappa\left(C_{i}\right)\right) \mu\left(H\left(\kappa\left(C_{i}\right)\right)\right)<c_{3} \alpha^{3} m n / \varepsilon^{2}
$$

for some constant $c_{3}>0$.

Finally, put

$$
\alpha=\frac{\varepsilon^{2}}{2} \sqrt{\frac{c \mu(\mathscr{Q})}{c_{\hat{3}} m}},
$$

where $c$ is the constant of Lemma 4.18. Note that $\alpha<\frac{1}{12}$ for any $n$ large enough (since $n \leq m$ ). Assume now that

$$
m t^{2}<\frac{c^{3} \varepsilon^{8} \mu(\mathscr{Q}) n^{2}}{4 c_{3}}
$$

and

$$
m<\frac{c^{5} \varepsilon^{8} \mu(\mathscr{Q}) n^{2}}{4 c_{3}}
$$

From (4.27) we have $\alpha n / t>1\left(c \varepsilon^{2}\right)$; therefore, Lemma 4.18 and (4.26) show that with probability greater than $1-\varepsilon$,

$$
c \varepsilon^{2} \alpha n\left(1-\varepsilon-\frac{1}{c^{2} \alpha \varepsilon n}\right) \mu(\mathscr{Q})<c_{3} \alpha^{3} m n / \varepsilon^{2},
$$

for $n$ large enough. From (4.28) we derive

$$
c \varepsilon^{2} \alpha n(1-2 \varepsilon) \mu(\mathscr{Q})<c_{3} \alpha^{3} m n / \varepsilon^{2},
$$

which gives a contradiction if, say, $\varepsilon<\min \left\{\varepsilon_{1}, \frac{1}{4}\right\}$. This implies that (4.27) or (4.28) has to be false. Since $\mu(\mathscr{Q})$ is bounded below by a positive constant, with probability greater than $1-\varepsilon$, we have $m t^{2}>c_{4} \varepsilon^{8} n^{2}$, for $c_{4}>0$ and $n$ large enough. Note that the condition $\varepsilon<\min \left\{\varepsilon_{1}, \frac{1}{4}\right\}$ can be relaxed by choosing $c_{4}$ small enough. 
Lemma 4.19. There exists a constant $c>0$ such that for any $\varepsilon(0<\varepsilon<1)$ and $n>1 / c$ a random set of $n$ points in $\mathscr{C}_{2}$ satisfies $m t^{2}>c \varepsilon^{8} n^{2}$ with probability greater than $1-\varepsilon$.

4.4.3. Summary of results and closing remarks. Let us recap the main results of this section (Lemmas 4.15 and 4.19) and state some immediate corollaries.

Lemma 4.20. Let $S$ be a faithful commutative semigroup and let $d>1$ be a positive integer and $\varepsilon$ any real $(0<\varepsilon<1)$. There exists a constant $c>0$ such that the following is true. Let $P$ be a random set of $n$ points in $\mathscr{C}_{d}$ and let $\Gamma$ be any storage scheme of size $m$ for the range searching problem $(S, \mathscr{Q}, P)$, where $\mathscr{Q}$ is the set of all slabs of fixed (appropriately chosen) width in $E^{d}$. Then if $n$ is large enough, with probability greater than $1-\varepsilon$, the time complexity $t=\max _{q \in \mathbb{Q}} t(P, \Gamma, q)$ satisfies the inequality $m t^{2}>c n^{2}$, for $d=2$, and $m t^{d}>c(n / \log n)^{d}$, for $d>2$. As a corollary, the worst case and average case time complexities satisfy

$$
t(n, m) \geq \bar{t}(n, m)=\Omega(n / \sqrt{m})
$$

for $d=2$, and

$$
t(n, m) \geq \bar{t}(n, m)=\Omega\left(\frac{n / \log n}{m^{1 / d}}\right)
$$

for $d \geq 3$.

Of course, these lower bounds also apply to simplex range searching, since a slab can always be clipped into a parallelotope without changing the nature of the problem, and a $d$-dimensional parallelotope can always be triangulated into at most $d$ ! simplices. We can therefore state our result in a more illustrative manner.

Theorem 4.21. Simplex range searching on $n$ points requires $\Omega(n / \sqrt{m})$ query time in two dimensions and $\Omega\left((n / \log n) / \mathrm{m}^{1 / d}\right)$ query time in any dimension $d \geq 3$, where $m$ denotes the amount of storage available. These bounds hold for a random point-set, and therefore are valid in the worst case as well as on the average.

As we mentioned in the introduction, simplex range searching on $n$ points in $d$-space can be performed in $O\left(n^{1-1 / d} \alpha(n)\right)$ query time and $O(n)$ storage, where $\alpha$ is a functional inverse of Ackermann's function (Chazelle and Welzl [5]). This upper bound, which holds in the arithmetic model, matches our lower bound very closely. On a random access machine supplied with linear storage, the best upper bound on the query time to date is $O(\sqrt{n} \log n)$ in 2space (Chazelle and Welzl [5]) and $O\left(n^{d(d-1) /(d(d-1)+1)+\varepsilon}\right)$ in $d$-space, for any $d \geq 3$ and any fixed $\varepsilon>0$ (Haussler and Welzl [11]). An interesting open problem is to bridge the gap in higher dimensions and generalize the upper bounds 
to general space-time trade-offs. Another intriguing question is to determine whether half-space queries are as hard as simplex queries.

\section{ACKNOWLEDGMENTS}

I would like to thank F. K. Chung, P. Erdös, R. L. Graham, A. M. Odlyzko, and E. Szemerédi for helpful discussions about Heilbronn's problem.

\section{REFERENCES}

1. M. Abramowitz and I. A. Stegun, Handbook of mathematical functions, Dover, New York, 1970.

2. A. V. Aho, J. E. Hopcroft, and J. D. Ullman, The design and analysis of computer algorithms, Addison-Wesley, Reading, MA, 1974.

3. W. A. Burkhard, M. L. Fredman, and D. J. Kleitman, Inherent complexity trade-offs for range query problems, Theor. Comput. Sci. 16 (1981), 279-290.

4. B. Chazelle, Lower bounds on the complexity of multidimensional searching, Proc. 27th Annual IEEE Sympos. on Foundations of Computer Science, IEEE, New York, 1986, pp. 87-96.

5. B. Chazelle and E. Welzl, Quasi-optimal range searching in spaces of finite VC-dimension, Discrete Comput. Geom. 4 (5) (1988).

6. R. Cole and C. K. Yap, Geometric retrieval problems, Inform. and Control 63 (1984), 39-57.

7. $\mathrm{H}$. Edelsbrunner and E. Welzl, Halfplanar range search in linear space and $O\left(n^{0.695}\right)$ query time, Inform. Process. Lett. 23 (1986), 289-293.

8. P. Erdös and J. Spencer, Probabilistic methods in combinatorics, Academic Press, New York, 1974.

9. M. L. Fredman, A lower bound on the complexity of orthogonal range queries, J. Assoc. Comput. Mach. 28 (1981), 696-705.

10. L L Lower bounds on the complexity of some optimal data structures, SIAM J. Comput. 10 (1981), 1-10.

11. D. Haussler and E. Welzl, Epsilon-nets and simplex range queries, Discrete Comput. Geom. 2 (1987), 127-151.

12. J. Komlós, E. Szemerédi, and J. Pintz, On Heilbronn's triangle problem, J. London Math. Soc. (2) 24 (1981), 385-396.

13. _ A lower bound for Heilbronn's problem, J. London Math. Soc. (2) 25 (1982), 13-24.

14. A. M. Macbeath, A compactness theorem for affine equivalence classes of convex regions, Canad. J. Math. 3 (1951), 54-61.

15. K. Mehlhorn, Data structures and algorithms 3: Multidimensional searching and computational geometry, Springer-Verlag, Berlin and New York, 1984.

16. W. O. J. Moser, Problems on extremal properties of a finite set of points, Discrete Geometry and Convexity, Ann. New York Acad. Sci. 440 (1985), 52-64.

17. L. A. Santaló, Integral geometry and geometric probability, Encyclopaedia Math. Appl. Vol. 1 (Gian-Carlo Rota, ed.), Addison-Wesley, Reading, MA, 1976.

18. D. E. Willard, Polygon retrieval, SIAM J. Comput. 11 (1982), 149-165.

19. A. C. Yao, On the complexity of maintaining partial sums, SIAM J. Comput. 14 (1985), $277-$ 288. 
20. F. F. Yao, $A$ 3-space partition and its applications, Proc. 15 th Annual ACM Sympos. on Theory of Comput., ACM, New York, 1983, pp. 258-263.

21. A. C. Yao and F. F. Yao, On computing the rank function for a set of vectors, Report No. UIUCDCS-R-75-699, Univ. of Illinois at Urbana-Champaign, 1975.

22. __ A general approach to d-dimensional geometric queries, Proc. 17th Annual ACM Sympos. on Theory of Comput., ACM, New York, 1985, pp. 163-168.

Department of Computer Science, Princeton University, Princeton, New Jersey 08544 